\title{
Evidence That the Central Nervous System Can Induce a Modification at the Neuromuscular Junction That Contributes to the Maintenance of a Behavioral Response
}

\author{
Kevin C. Hoy, ${ }^{1}{ }^{\circledR}$ Misty M. Strain, ${ }^{2}$ Joel D. Turtle, ${ }^{3}$ Kuan H. Lee, ${ }^{3}{ }^{\circledR}$ J. Russell Huie, ${ }^{4}$ John J. Hartman, ${ }^{3}$ \\ ${ }^{\circledR}$ Megan M. Tarbet, ${ }^{3}$ Mark L. Harlow, ${ }^{5}{ }^{\circledR}$ David S.K. Magnuson, ${ }^{6}$ and ${ }^{\circledR}$ James W. Grau ${ }^{3}$ \\ ${ }^{1}$ Case Comprehensive Cancer Center/Case Western Reserve School of Medicine, Cleveland, Ohio 44106, ${ }^{2}$ U.S. Army Institute of Surgical Research, \\ JBSA Fort Sam Houston, Houston, Texas $78234,{ }^{3}$ Department of Psychological and Brain Sciences, Texas A\&M University, College Station, Texas \\ $77843,{ }^{4}$ Department of Neuroscience, University of California San Francisco, San Francisco, California 94110, ${ }^{5}$ Department of Biology, Texas A\&M \\ University, College Station, Texas 77843, and ${ }^{6}$ Department of Neurological Surgery, University of Louisville, Louisville, Kentucky 40202
}

Neurons within the spinal cord are sensitive to environmental relations and can bring about a behavioral modification without input from the brain. For example, rats that have undergone a thoracic (T2) transection can learn to maintain a hind leg in a flexed position to minimize exposure to a noxious electrical stimulation (shock). Inactivating neurons within the spinal cord with lidocaine, or cutting communication between the spinal cord and the periphery (sciatic transection), eliminates the capacity to learn, which implies that it depends on spinal neurons. Here we show that these manipulations have no effect on the maintenance of the learned response, which implicates a peripheral process. EMG showed that learning augments the muscular response evoked by motoneuron output and that this effect survives a sciatic transection. Quantitative fluorescent imaging revealed that training brings about an increase in the area and intensity of ACh receptor labeling at the neuromuscular junction (NMJ). It is hypothesized that efferent motoneuron output, in conjunction with electrical stimulation of the tibialis anterior muscle, strengthens the connection at the NMJ in a Hebbian manner. Supporting this, paired stimulation of the efferent nerve and tibialis anterior generated an increase in flexion duration and augmented the evoked electrical response without input from the spinal cord. Evidence is presented that glutamatergic signaling contributes to plasticity at the NMJ. Labeling for vesicular glutamate transporter is evident at the motor endplate. Intramuscular application of an NMDAR antagonist blocked the acquisition/maintenance of the learned response and the strengthening of the evoked electrical response.

Key words: learning; memory; muscle; neuromuscular junction; NMDA receptor; peripheral

Significance Statement

The neuromuscular junction (NMJ) is designed to faithfully elicit a muscular contraction in response to neural input. From this perspective, encoding environmental relations (learning) and the maintenance of a behavioral modification over time (memory) are assumed to reflect only modifications upstream from the NMJ, within the CNS. The current results challenge this view. Rats were trained to maintain a hind leg in a flexed position to avoid noxious stimulation. As expected, treatments that inhibit activity within the CNS, or disrupt peripheral communication, prevented learning. These manipulations did not affect the maintenance of the acquired response. The results imply that a peripheral modification at the NMJ contributes to the maintenance of the learned response.

Received Nov. 12, 2019; revised 0ct. 13, 2020; accepted 0ct. 14, 2020.

Author contributions: K.C.H., M.M.S., J.D.T., K.H.L., J.R.H., D.S.K.M., and J.W.G. designed research; K.C.H., M.M.S., J.D.T., K.H.L., J.R.H., J.J.H., and M.M.T. performed research; K.C.H., M.M.S., J.D.T., K.H.L., J.J.H., M.M.T., M.L.H., and J.W.G. analyzed data; K.C.H. wrote the first draft of the paper; M.M.S., J.D.T., J.R.H., M.M.T., M.L.H., D.S.K.M., and J.W.G. edited the paper.

This work was supported by the Mary Tucker Currie Professorship to J.W.G. The Olympus FV1000 confocal microscope acquisition, performed at the Microscopy and Imaging Center at Texas A\&M University, was supported by the Office of the Vice President for Research. We thank Rachel Baine, Paris Bean, Jacob Davis,
Gizelle Fauss, Kie Huang, Kelsey Hudson, Travis Johnston, Mathew Lee, Jeffrey Petruska, Josh Reynolds, and Wes Thompson for help and input.

The authors declare no competing financial interests.

Correspondence should be addressed to James W. Grau at j-grau@tamu.edu.

https://doi.org/10.1523/JNEUROSCI.2683-19.2020

Copyright $\odot 2020$ the authors 


\section{Introduction}

In vertebrates, it is generally assumed that learning about new environmental relations (acquisition), and its retention over time (maintenance), are mediated by the CNS. From this perspective, ongoing efferent motoneuron output drives peripheral processes that maintain a behavioral response. Implicit here is an assumed rigidity at the neural muscular interface, guaranteeing that a neural output faithfully elicits a behavioral response (Wood and Slater, 2001). In adult vertebrates, this interface depends on the release of acetylcholine (ACh) from the efferent motoneuron and the activation of the ACh receptors (AChR) that form the neuromuscular junction (NMJ) (Sanes and Lichtman, 1999, 2001).

The current study shows that learning can engage a peripheral modification at the NMJ that contributes to the maintenance of a behavioral response. The findings emerged from work examining the neurobiological mechanisms involved in a form of learning mediated by neurons within the spinal cord. In this paradigm, communication with the brain is cut by means of an upper thoracic (T2) transection (Grau et al., 1998, 2006; Grau, 2014). Electrical stimulation (shock) is applied to the tibialis anterior muscle of one hind leg, eliciting a flexion response (Fig. $1 A$ ). If stimulation is given whenever the leg is extended (controllable stimulation), animals exhibit an increase in flexion duration that minimizes net shock exposure. These observations imply that the behavioral modification depends on the relation between a response (leg position) and an environmental outcome (noxious stimulation), a form of learning known as instrumental conditioning. Here, the maintenance of a flexion response over time represents a kind of behavioral memory.

To establish that learning depends on spinal cord neurons, the $\mathrm{Na}^{+}$channel blocker lidocaine was delivered onto the lumbosacral spinal cord through an intrathecal (i.t.) catheter before training (Crown et al., 2002a). Lidocaine blocked learning. Cutting CNS communication with the periphery by transecting the sciatic nerve that innervates the tibialis anterior muscle had the same effect. Further work has related spinally mediated plasticity to signal pathways implicated in brain-dependent learning and memory (Ji et al., 2003; Joynes et al., 2004; Grau, 2014). It was hypothesized that these cellular processes bring about a lasting alteration (a memory-like effect) that increases the motoneuron output, maintaining the flexion response (Fig. 1B).

The present study was motivated by an observation that challenged this conceptual framework. Because excitatory neural transmission within the spinal cord depends on glutamate and the activation of the ionotropic AMPA receptor (AMPAR), it is not surprising that intrathecal application of the AMPAR antagonist CNQX blocks spinal learning (Hoy et al., 2013). If learning brings about an intraspinal modification that drives the motoneuron output, intrathecal CNQX should also disrupt the maintenance of the learned response. Yet, when CNQX was applied intrathecally after animals had received 25 min of training, it had no immediate effect (Hoy et al., 2013). This observation motivated the first experiment reported here, which tested whether inactivating neural activity with the anesthetic lidocaine disrupts the maintenance of the learned response. Lidocaine (i.t.) had the same effect as CNQX: it blocked learning but had no effect when given after the behavioral modification was acquired. Likewise, cutting the sciatic nerve had no effect after the learned response was established. These novel findings imply that the CNS can induce a peripheral alteration that helps maintain the behavioral modification over time. We reinforce this conclusion with evidence that learning amplifies electrical activity within the muscle and that this effect also survives a sciatic transection. Fluorescent imaging related the potentiation of the flexion response to an increase in the density of the AChR at the NMJ. It is suggested that motoneuron output, in conjunction with stimulation of the tibialis anterior muscle, strengthens the connection at the NMJ in a Hebbian manner. Supporting this, paired stimulation of the efferent nerve and tibialis anterior muscle led to an increase in flexion duration and amplified the evoked electrical response without input from the spinal cord. Evidence is presented that the activation of the NMDA receptor (NMDAR) at the NMJ contributes to the development and maintenance of this peripheral memory.

\section{Materials and Methods}

Subjects

Male Sprague Dawley rats (100-120 d old, 300-450 g) were obtained from Envigo and acclimated for at least $7 \mathrm{~d}$ before experimentation. Animals were dual-housed with water and food ad libitum and maintained on a $12 \mathrm{~h}$ light-dark cycle. Behavioral testing and surgeries were performed during the light portion of the cycle. All experiments were conducted in accordance with National Institutes of Health standards for the care and use of laboratory animals (National Institutes of Health publication no. 80-23), and were approved by the Institutional Animal Care and Use Committee at Texas A\&M University. Every effort was made to minimize suffering and limit the number of animals used.

\section{Surgery and drug infusion}

Spinal Transection. All rats received a complete spinal transection just rostral to the second thoracic (T2) vertebra as described by Hoy et al. (2013). Animals were anesthetized with isoflurane, the T2 vertebra was located by palpation, and two incisions were made rostrocaudal to the vertebra. The spinal cord was exposed just rostral to T2 by removing tissue along the dorsal aspect and was then transected by thermal cautery. Sham-operated animals underwent the same procedure, except the spinal cord was not transected. Animals were injected with $0.9 \%$ saline (4 ml, i.p.) immediately following surgery and twice daily for the duration of the experiments. Rats were allowed to recover overnight (18-24 h) in a temperature-controlled room $\left(25^{\circ} \mathrm{C}\right)$ with water and food ad libitum. During recovery, the animal's legs were secured in a natural position using porous tape. Bladders were expressed twice daily and immediately before any experimental procedures. When experimentation was complete, animals were killed with a lethal dose of pentobarbital $(100 \mathrm{mg} / \mathrm{kg}$, i.p.). The surgical transections were verified by (1) visual inspection of the transection site during surgery, (2) observing behavior during the recovery period to confirm complete paralysis and a lack of vocalization to leg shock, and (3) postmortem examination of the spinal cord in a random sample of subjects.

Intrathecal catherization. A catheter was inserted into the intrathecal space as described in Crown et al. (2002b). Briefly, a $25 \mathrm{~cm}$ segment of PE-10 tubing with a $0.23 \mathrm{~cm}$ (diameter) stainless-steel guide wire (SWGX-090; Small Parts) was threaded caudally $9 \mathrm{~cm}$ into the subarachnoid space at T2, so that the tubing laid along the dorsal surface of the L4-L5 spinal segments. The wire was then removed and the tubing was secured to the caudal vertebra with adhesive (Superglue).

Nerve dissection and electrode placement. The sciatic nerve was exposed by a transverse incision across the thigh to reveal the biceps femoris and vastus lateralus muscles. The sciatic nerve was exposed using blunt dissection along the fascial plane between the two muscles. To cut the sciatic nerve, a suture was threaded underneath the exposed nerve. The nerve was gently lifted and transected using scissors. In experiments using sciatic stimulation, a nerve cuff with two platinum stimulating leads (Plexon) was positioned near the insertion of the sciatic into the spinal column. To expose the common peroneal nerve, an incision was made along the lateral side of the lower limb exposing the proximal tibialis anterior muscle. The fascia surrounding the nerve was dissected using blunt dissection. The exposed peroneal nerve was then fitted with a stimulating electrode made from two thin (diameter: 
A

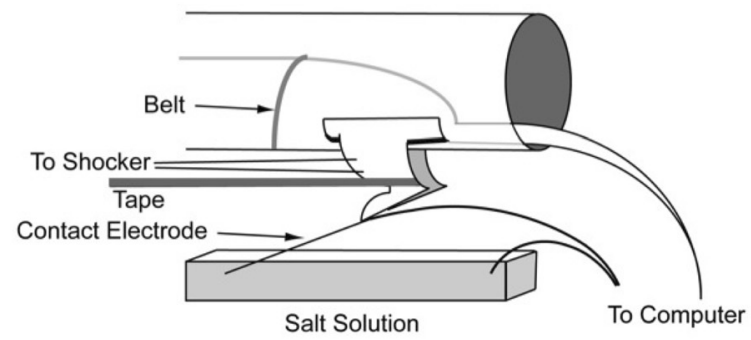

B

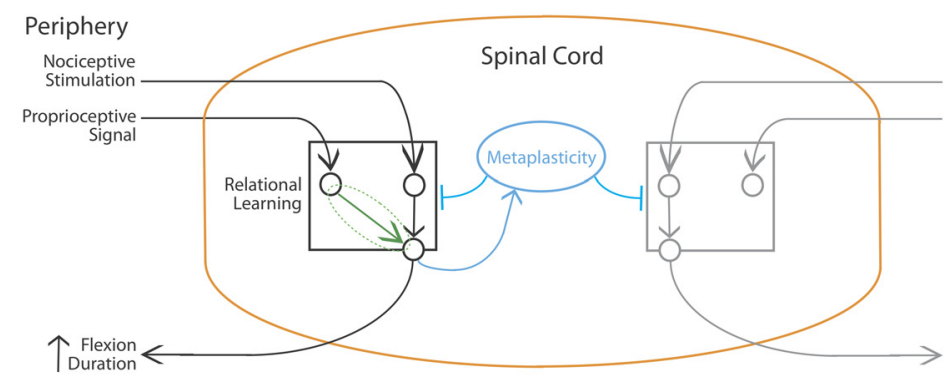

Figure 1. The apparatus used for instrumental training and a model of spinal learning. $\boldsymbol{A}$, Shock is applied to the tibialis anterior muscle, and leg position is monitored by means of a rod (contact electrode) that is taped to the rat's paw. When the leg is extended, the rod touches the underlying salt solution, completing a circuit that is monitored by a computer. Rats in the Master condition receive shock to the muscle whenever the hind leg is extended (response-contingent). In spinally transected rats, this brings about an increase in flexion duration that reduces net shock exposure. Animals that are experimentally coupled (Yoked) to the Master rats, and receive shock at the same time, independent of leg position (noncontingent), do not exhibit an increase in flexion duration (the index of learning). $\boldsymbol{B}$, Prior work suggested that learning depends on the relationship between the onset of nociceptive stimulation and an index (proprioceptive signal) of limb position (Grau et al., 2012; Grau, 2014). Learning was thought to establish an intraspinal modification (dashed ellipse) that drives the motoneuron output (green line). Relational learning also appears to engage processes (blue) that have a lasting effect on the capacity to learn, a form of metaplasticity (Ferguson et al., 2008; Grau et al., 2014). For example, experience with response-contingent stimulation enables subsequent learning, both on the pretrained and contralateral limb (gray lines and box).

$0.27 \mathrm{~mm}$ ) wires $25 \mathrm{~cm}$ in length. The last $0.5 \mathrm{~cm}$ of each wire was stripped of insulation. The two wires were then shaped to form small hooks spaced $2 \mathrm{~mm}$ apart (el-Bohy et al., 1998). The skin was sutured closed and the animal was placed in a warm room until training.

Excision of the L3-S3 spinal cord. To prepare the L3-S3 region for removal, a laminectomy was performed at the T13-L2 vertebrae to expose the tissue before the T2 transection. The next day, the incision was opened, and the tissue was flooded with saline to maintain hydration. Twenty-five minutes later, the exposed tissue was transected at L3 and S3 with a cautery device. A razor blade was then used to make longitudinal cuts along the sides of the spinal cord. Finally, the isolated spinal tissue was removed with tweezers. The inside of the vertebral column was cleaned with a cotton swab to ensure that the desired length of the spinal cord was completely removed. Removing the spinal cord took $\sim 1 \mathrm{~min}$.

Tibialis anterior muscle injections. A $27 \mathrm{G}$ needle tip was inserted into the tibialis anterior muscle before training. The $27 \mathrm{G}$ needle tip was connected to a $29-\mathrm{cm}$-long PE-50 tube, which was attached to a $1 \mathrm{ml}$ syringe for drug delivery.

\section{Behavioral assays}

Tail-flick test. Thermal nociceptive thresholds were assessed using a radiant heat tail flick device (see Grau, 1984). Animals were loosely restrained in opaque Plexiglas tubes $(23.5 \mathrm{~cm} \times 8 \mathrm{~cm}$ ), and room temperature was maintained at $26.7^{\circ} \mathrm{C}$. Lateral tail movements exceeding $0.5 \mathrm{~cm}$ terminated the heat source, and latency was recorded to the nearest $0.01 \mathrm{~s}$. If an animal did not respond to radiant heat within $8 \mathrm{~s}$, the test trial was terminated to prevent tissue damage.

Instrumental Training Apparatus. Instrumental training was conducted as described by Grau et al. (1998). Briefly, rats were loosely restrained in tubes with slots that allowed the hind legs to hang freely (Fig. 1A). Two electrodes were then inserted into one hindleg. The first electrode was constructed of stainless-steel wire $(0.255 \mathrm{~mm}$ diameter $)$ and was inserted through the skin over the tibia, $1.5 \mathrm{~cm}$ from the tarsus. The second was made of fine wire $(0.127 \mathrm{~mm}$ diameter, magnet wire single Beldsol) and was inserted perpendicular to the leg, through the body of the tibialis anterior muscle, $1.7 \mathrm{~cm}$ above the first electrode. Shock was delivered using a BRS/LVE (Laurel) AC shock generator (model SG903). Leg position was monitored by taping a $7 \mathrm{~cm}$ stainless-steel rod $(0.46 \mathrm{~mm}$ diameter) to the rat's paw. To prevent current flow through the rod to the rat, the portion of the rod that contacted the rat's paw was insulated with heat shrink tubing. A fine wire $(0.26 \mathrm{~mm}$ diameter; $20.0 \mathrm{~cm}$ length) was attached to the end of the foot electrode and was connected to a digital input monitored by a Macintosh computer. A rectangular plastic dish containing a solution of $\mathrm{NaCl}$ was placed $\sim 7.5 \mathrm{~cm}$ below the restraining tube and connected to ground. Whenever the contact electrode touched the underlying salt solution, it completed the circuit, enabling the presentation of response-contingent stimulation.
Instrumental training procedure. To equate flexion force across animals before training, a monofilament plastic line was attached to the rat's ankle and connected to a strain gauge (Baumbauer et al., 2009). A $300 \mathrm{~ms}(60 \mathrm{~Hz}$, sinusoidal) shock was then applied, and intensity was adjusted to elicit a flexion force of $0.4 \mathrm{~N}$. The monofilament was then removed, and three $0.15 \mathrm{~s}$ leg-shocks were applied to establish a resting position of the leg. The level of underlying saline solution was then adjusted so that the tip of the contact electrode was submerged by $4 \mathrm{~mm}$. Response-contingent (controllable) shock was applied by administering electrical stimulation to the tibialis anterior muscle whenever the contact electrode touched the underlying salt solution. To assess the effect of shock per se, some animals were experimentally coupled (Yoked) to animals that had behavioral control (Master) and were given shock at the same time, but independent of leg position. Unshocked controls were set up in a similar manner, but never received shock.

A similar procedure was used to evaluate whether paired stimulation of the peroneal nerve and tibialis anterior muscle can support learning. Electrodes were implanted in tibialis anterior muscle, and the level of the underlying solution was adjusted as described above. In addition, the common peroneal nerve was exposed, and two hook electrodes were attached $2 \mathrm{~mm}$ apart. The proximal hook wire served as the positive lead. Animals received stimulation to the tibialis anterior muscle and peroneal, the tibialis anterior muscle alone, or the peroneal alone. In each case, stimulation was applied whenever the contact electrode touched the underlying salt solution and animals were tested for $30 \mathrm{~min}$.

Instrumental performance was assessed over time by breaking the training/testing session into $1 \mathrm{~min}$ bins. Within each, we computed time in solution, response number, and response duration. Response duration was derived from time in solution and response number using the following equation: Response Duration $_{\mathrm{i}}=\left(60 \mathrm{~s}-\right.$ Time in solution $\left._{\mathrm{i}}\right) /$ (Response Number $r_{i}+1$ ), where $i$ is the current time bin. Response Rate indicates the number of responses per min (for further details, see Grau et al., 1998).

Pavlovian training procedure. For Pavlovian training, rats were given AC stimulation $(0.6 \mathrm{~mA}$, sinusoidal $)$ to the tibialis anterior muscle and DC stimulation ( $4 \mathrm{~V}, 0.5 \mathrm{~ms}$ pulses, unipolar, $100 \mathrm{~Hz}$ ) to the peroneal nerve. The stimuli were presented for $80 \mathrm{~ms}$, and each was given 180 times. Half the rats received the stimuli simultaneously in a paired manner, on a variable schedule with the mean interstimulus interval of $2 \mathrm{~s}$ $(0.5 \mathrm{~Hz} ; 0.2-3.8 \mathrm{~s})$ or $10 \mathrm{~s}(0.1 \mathrm{~Hz} ; 0.2-19.8 \mathrm{~s})$. The remaining animals experienced the AC and DC stimuli in an explicitly unpaired (alternating) manner, with the frequency of the AC stimulation matched to the paired condition at 0.5 or $0.1 \mathrm{~Hz}$.

A kinematic analysis was performed to evaluate the effect of stimulation on leg position. Animals had their ankles, knee, and hip marked with a black sharpie. A video camera was placed lateral to the leg. Tracker version 4.87 was used to trace the angle of the limb at baseline 
and after 30,60,90,120,150, and 180 stimulations. Limb angle was assessed again $30 \mathrm{~min}$ after the end of training.

$E M G$

EMG apparatus. Two thin wire electrodes (diameter $0.27 \mathrm{~mm}$ ) were inserted into the tibialis anterior muscle for EMG recording. One electrode (positive lead) was inserted into the body of the muscle below the stimulating wire electrode required for instrumental learning. The other electrode (negative lead) was placed $1 \mathrm{~cm}$ distal to the positive lead electrode. A third wire electrode was inserted into the skin on the ventral aspect of the leg just superior to the ankle (ground).

Nerve stimulation was delivered using a Grass Stimulator (model S9) connected to a Plexon platinum nerve cuff. Sciatic stimulation was delivered as $10 \mathrm{~ms}$ bursts of shock with an interstimulus interval of $10 \mathrm{~s}$ at a voltage of 1 or $2 \mathrm{~V}$. Because subsequent analyses revealed a ceiling effect when the higher $(2 \mathrm{~V})$ intensity was applied, we focused on the response elicited by the moderate $(1 \mathrm{~V})$ stimulus. A Plexon headstage and amplifier (PBX-633) were connected to a National Instruments digital/analog converter (NI SCB-68 DAQ), and data were recorded using a Windows computer and Plexon Recorder software. Data were filtered online using a $500 \mathrm{~Hz}$ low-pass filter and a $60 \mathrm{~Hz}$ notch filter to remove line noise. The same apparatus was used to measure the EMG response to peroneal stimulation.

A custom-built Faraday cage enclosed the rat as well as all recording equipment. In addition, a custom relay protection system prevented high-voltage shocks from damaging EMG recording equipment. Two QPDT relays were wired to shunt current originating from the shock generator directly to ground unless shock was currently being applied, isolating the EMG recording circuit from the AC shock (instrumental learning) circuit.

EMG analysis. Recordings were imported into MATLAB for rectification and analysis. For activity during training, the EMG response was normalized to baseline activity recorded after the first burst of three AC shocks and before the initiation of training. For studies examining EMG activity in response to nerve stimulation, EMG traces were rectified and aligned using an automated custom MATLAB script. Data were collected for $100 \mathrm{~ms}$, starting $10 \mathrm{~ms}$ before nerve stimulation.

\section{Fluorescent imaging}

Muscle collection/preparation. At the end of training, rats were removed from the apparatus, and the tibialis anterior muscle was extracted from each hind leg under pentobarbital anesthesia. Muscles were placed in $1 \%$ PFA in potassium PBS (KPBS) for $1 \mathrm{~h}$ and cryoprotected in $20 \%$ sucrose in KPBS overnight. The tissue was embedded in an optimal cutting temperature medium (Tissu-Tek, Sakura Finetek) and frozen in liquid nitrogen cooled acetone; $10 \mu \mathrm{m}$ cross sections were cut, affixed to positively charged slides, and stored at $-80^{\circ} \mathrm{C}$.

Immunohistochemistry. Slides were equilibrated in KPBS, washed in $0.1 \mathrm{M}$ glycine in KPBS for $1 \mathrm{~h}$ at room temperature, and extracted with $0.5 \%$ Triton in KPBS for $15 \mathrm{~min}$ on ice. The slides were washed in $1 \times$ KPBS 3 times for $10 \mathrm{~min}$ each. Tissue was blocked in $1 \%$ gelatin KPBS for $1 \mathrm{~h}$ in a black humidified box. Primary antibody was placed onto tissue mixed with PBS containing glucose. The AChR was labeled using $\alpha$-bungarotoxin conjugated with AlexaFluor-488 dye (1:200; Invitrogen; B-13422). Tissue was allowed to incubate overnight at room temperature. On the second day, the slides were washed 3 times for $15 \mathrm{~min}$ in PBS containing glucose $+0.1 \%$ Tween- 20 and treated with the secondary antibodies for 2-3 $\mathrm{h}$ in humidified boxes lightly shaken using an orbital shaker. Slides were washed 3 times for $15 \mathrm{~min}$ in PBS containing glucose $+0.1 \%$ Tween- 20 and washed with DI $\mathrm{ddH}_{2} \mathrm{O}$ several times for 3-5 s. Slides were then mounted using Prolong Gold antifade reagent and coverslipped with 1.5 coverslips sealed with clear nail polish.

For analysis of VAChT and VGluTs 1 and 2, a similar wash and stain protocol was used as above, with the following changes. Mouse monoclonal antibodies for VGluT1 (1:100; NeuroMab; 75-066; RRID:AB_ 2187693) and VGluT2 (1:100; NeuroMab; 75-067; RRID:AB_2239153) were labeled in separate experiments. Rabbit polyclonal was used to label VAChT (1:100; Abcam; ab68986; RRID:AB_1143827). Fluorescenttagged secondary antibodies included Pacific Blue Goat anti-mouse
(Invitrogen; P31582; RRID:AB_10374586) and Alexa-488 Goat anti-rabbit (Invitrogen; A-11 034; RRID:AB_2576217). Alexa-594 a-bungarotoxin (Invitrogen; B-13 423; CID: 44264212) was used to label AChRs.

Confocal microscopy. Labeling was assessed using an Olympus FV300 confocal microscope using a $60 \times$ Plan/Apo 1.42 oil objective. The Olympus Fluoview version 5.0 software was used for imaging. For each pair of trained and untrained tissue, the PMT and offset were consistent throughout imaging. A medium speed (5.36 s) scan was used, and image size was set to $1024 \times 1024$. A Kalman filter was also used. Scan depth was determined by $\alpha$-bungarotoxin labeling. The appearance/disappearance of the ACh signal was used to set the upper and lower bounds for the $z$ axis (average stack height $=21.2 \pm 0.39$ ). A 488 laser (blue light) was emitted onto the Alexa-488 dye giving green images. The Alexa-546 was excited by a $543 \mathrm{~nm}$ laser, eliciting a red image. Images were saved in a merged 24 bit image format and a 16 bit grayscale format for each of the channels. The 16 bit grayscale images were used for analysis.

VGluT1,2/VAChT labeling was assessed using an Olympus FV1000 confocal microscope equipped with a UPLSAPO $100 \times / 1.4$ oil immersion objective. The Olympus FV10-ASW software was used for imaging. A $4 \mathrm{~s}$ scan speed was used, and image size and scan depth were determined by $\alpha$-bungarotoxin labeling. Imaging lasers used included $405 \mathrm{~nm}$ for the Pacific Blue, $488 \mathrm{~nm}$ for Alexa-488, and $543 \mathrm{~nm}$ for Alexa-555. Images were saved and analyzed in a 16 bit grayscale format for each of the channels using ImageJ software. Maximum intensity projections of $\sim 5 \mu \mathrm{m}$ in depth were then assessed for the presence and colocalization of VGlut1,2 transporters with VAChT adjacent to the $\alpha$-bungarotoxin labeled NMJ.

Image analysis. Quantitative assessment of AChR labeling was performed using the ImageJ software package. After the NMJs within a muscle section were identified, 10 were randomly selected for analysis. For the AChR, a stack was formed from the $1024 \times 1024$ grayscale images, and signal intensity for the $\alpha$-bungarotoxin was measured using the integrated density function and mean gray value. In addition, for each stack, we outlined a smaller $(200 \times 320$ pixel $)$ ROI that contained the AChR and reran the analyses. In all cases, scan parameters were kept constant across key variables (trained vs untrained legs and subjects). The following measures were used in our analyses:

Pixel intensity: the 16 bit value for pixels that exceeded threshold;

Mean depth: the average number of scans required within a stack to encompass the AChR;

Mean area: the average number of pixels that met threshold within one scan;

Net intensity: Mean Area $\times$ Pixel Intensity summed across scans; and

Maximum scan intensity: the maximum Mean Area $\times$ Pixel Intensity value within a stack.

\section{Experimental design and statistics}

All experiments used balanced factorial designs that are specified below, along with the number of animals per condition $(n)$. Animals were randomly assigned to groups with an equal $n$ per condition. The number of animals used in each experiment was sufficient to resolve a large effect size $(d>1.4)$ (Cohen, 1988). Care was taken to assure that the individuals collecting data were blinded to experimental condition. Data were analyzed using ANOVA or ANCOVA (SuperANOVA, Abacus Concepts). Alpha values of $\leq 0.05$ were considered statistically significant. Post hoc comparisons at particular time points were made using Student's $t$ test with a Bonferroni correction to maintain the overall error rate at 0.05 . Group performance was compared using Duncan's new multiple range test, which maintains the error rate at 0.05 for a family of contrasts.

\section{Results}

Intrathecal application of lidocaine blocks acquisition, but not the maintenance, of a learned response

Prior work has established that spinally transected rats can learn to maintain a hind leg in a flexed position to minimize exposure to noxious electrical stimulation (Grau et al., 1998, 2006). We 
A

General Design

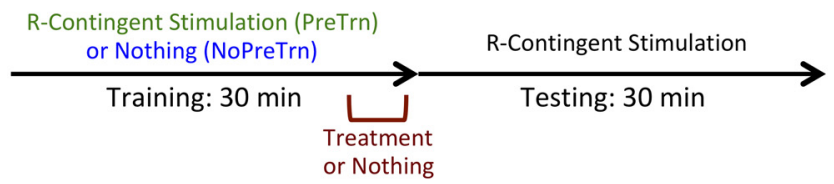

C



E

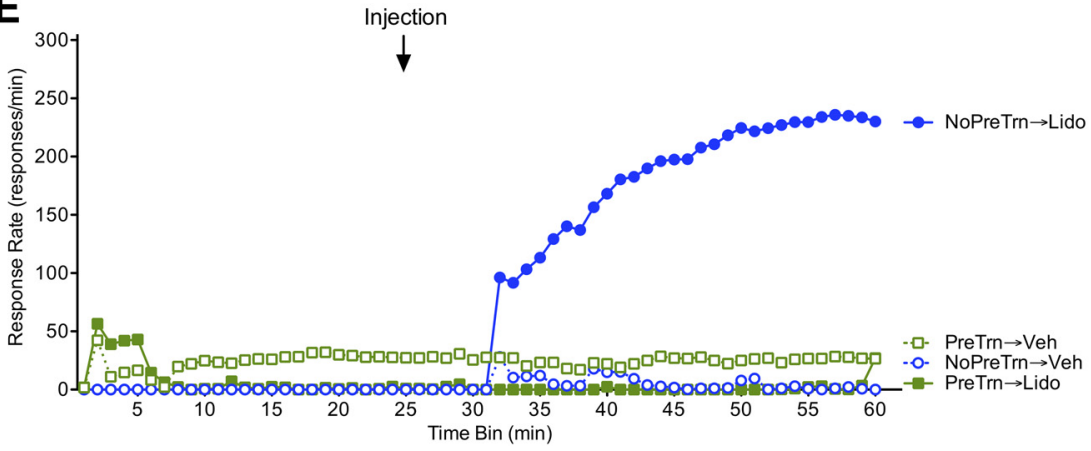

B

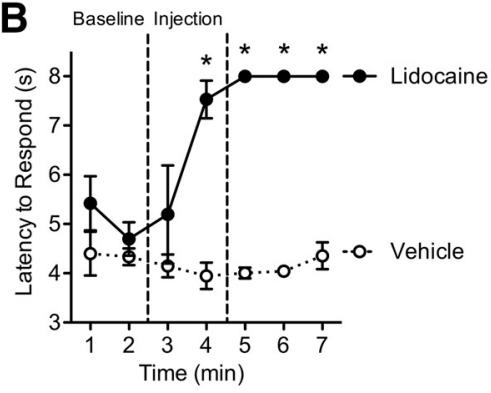

D

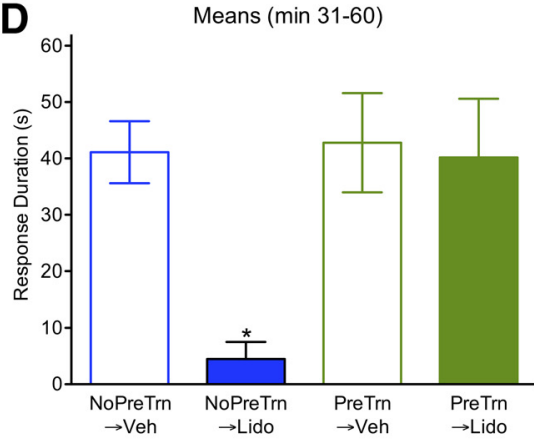

F

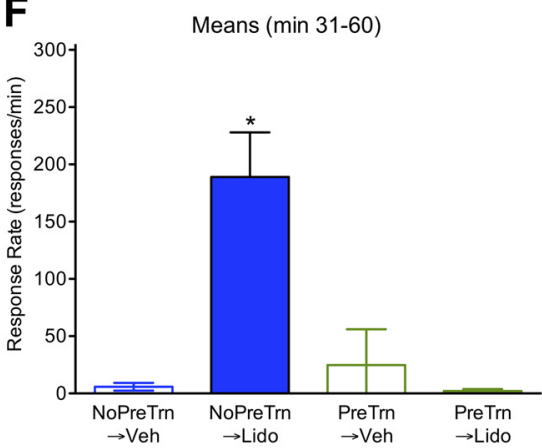

Figure 2. Inactivating neurons within the spinal cord using the anesthetic lidocaine blocks the acquisition, but not the maintenance, of a learned response in spinally transected rats. $\boldsymbol{A}$, The design used to assess whether experimental treatments affect the maintenance of the learned response. $\boldsymbol{B}$, Intrathecal lidocaine blocks the performance of a spinal reflex, tail withdrawal from a noxious thermal stimulus, within 1 min of application $(n=4)$. C, Average response duration over time during training and testing. Rats were given 30 min of training (PreTrn) or nothing (NoPreTrn) and injected intrathecally with lidocaine (Lido) or vehicle (Veh) at $25 \mathrm{~min}(n=6)$. Rats that were not pretrained and received the vehicle (NoPreTrn $\rightarrow$ Veh) exhibited an increase in flexion duration. This learning was blocked by intrathecal lidocaine (NoPreTrn $\rightarrow$ Lido). Lidocaine had no effect on the performance of the learned response in pretrained rats (PreTrn $\rightarrow$ Lido). $\boldsymbol{D}$, Mean performance after drug infusion (min 31-60). $\boldsymbol{E}$, Acquisition of a learned response is accompanied by a reduction in rate of responding. Rats that received lidocaine before the onset of training (NoPreTrn $\rightarrow$ Lido) failed to learn and exhibited the highest rate of responding. Lines indicate the average number of responses made per minute over time during training and testing. $\boldsymbol{F}$, Mean response rate after drug infusion (min 31-60). Error bars indicate SEM. Asterisks indicate $p<0.05$.

had naturally assumed that learning brings about an alteration within the spinal cord that increases the efferent motor output to the tibialis anterior muscle, thereby maintaining the stimulated leg in a flexed position. If this is true, then pharmacological treatments that interfere with neural transmission within the spinal cord should disrupt the maintenance of the learned response. Contrary to our working hypothesis, we report here that inactivating neurons within the spinal cord with lidocaine has no effect on the maintenance of the learned response.

In prior studies, we have shown that the intrathecal lidocaine procedure used here inhibits spinally mediated learning (Crown et al., 2002a), the induction of maladaptive plasticity (Joynes et al., 2003), and brain-dependent responses to nociceptive stimulation in contused rats (Davis et al., 2020). To further verify the effectiveness of this treatment, we tested its effect on the performance of a spinally mediated reflex [tail withdrawal from a noxious thermal stimulus (tail-flick test)]. Animals were placed in restraining tubes for $5 \mathrm{~min}$ to acclimate to the testing room. Next, the latency to exhibit a tail-flick response to radiant heat was assessed 2 times at 1 min intervals. After baseline testing, lidocaine $(20 \mu \mathrm{l}, 40 \%)$ or saline was slowly infused over a $2 \mathrm{~min}$ period, during which two additional tail-flick tests were performed. Three additional tests were conducted at 1 min intervals. If an animal did not respond within $8 \mathrm{~s}$, the test trial was terminated. The full design involved two groups (Lidocaine vs Vehicle) with repeated measures (tail-flick testing) $(n=4)$.

Tail-flick latencies did not differ before drug treatment $\left(F_{(1,6)}=1.98, p>0.05\right)$. Administration of lidocaine caused a rapid inhibition of the tail-withdrawal response that was evident within 1-2 min (Fig. 2B). To control for variation in baseline reactivity, an ANCOVA was performed with the mean baseline score serving as the covariate. The analysis yielded a significant effect of lidocaine $\left(F_{(1,5)}=101.08, p<0.0002\right)$ and a significant time $\times$ lidocaine interaction $\left(F_{(4,20)}=3.21, p<0.0346\right)$. Post hoc 
comparisons of the mean response latency at each time point using a Student's $t$ test with a Bonferroni correction showed that lidocaine had a significant effect within $1 \mathrm{~min}$ of application $(p<0.05)$.

Having established that intrathecal lidocaine blocks the performance of a spinal reflex within 2 min of application, we tested whether this drug treatment affects the performance of a learned response. Rats were set up in an identical manner, and then half (PreTrn) received 30 min of training with response-contingent legshock (Fig. 2A). Half the pretrained animals (PreTrn $\rightarrow$ Lido) had lidocaine (i.t.; $20 \mu \mathrm{l}, 40 \%$ ) slowly infused after $25 \mathrm{~min}$ of training. The other pretrained rats (PreTrn $\rightarrow$ Veh) were treated with vehicle. To verify that intrathecal lidocaine disrupts the acquisition of an instrumental response, the remaining animals received no pretraining (NoPreTrn) before drug delivery. Five minutes after they received lidocaine $($ NoPreTrn $\rightarrow$ Lido) or vehicle (NoPreTrn $\rightarrow$ Veh), they were tested for $30 \mathrm{~min}$ with response-contingent shock. The full design involved a 2 (PreTrn or NoPreTrn $) \times 2$ (Lido or Veh) factorial $(n=6)$. Data from the training (min 1-30) and test phases (min 31-60) were analyzed separately, with time bin serving as a withinsubjects variable.

Animals that were pretrained (PreTrn) with response-contingent shock exhibited an increase in flexion duration across the 30 min of training $\left(F_{(29,290)}=3.09, p<0.0001\right)$, and performance during pretraining did not differ across groups (both $F$ values $<$ 1.0, $p>0.05$ ) (Fig. 2C). After the intrathecal injection, rats that received no pretraining and vehicle (NoPreTrn $\rightarrow$ Veh) exhibited an increase in flexion duration indicative of learning. Application of lidocaine blocked learning in rats that were not pretrained (NoPreTrn $\rightarrow$ Lido), but had no effect on the performance of the learned response in animals that were previously trained (PreTrn $\rightarrow$ Lido). An ANOVA conducted on the data from the 30 min test session confirmed that the main effects of drug treatment and time were significant (both $F$ values $>2.53, p<0.05$ ). Further, the main effect of drug treatment depended on pretraining condition $\left(F_{(1,20)}=4.63, p=0.0439\right)$. In addition, the interactions between time, pretraining condition, drug treatment, and their combination were significant (all $F$ values $>1.79, p<0.05$ ). Post hoc comparisons of the group means (Fig. 2D), collapsed across the last $30 \mathrm{~min}$ of testing, showed that the untrained lidocaine group (NoPreTrn $\rightarrow$ Lido) was significantly different from all other groups $(p<0.05)$. No other comparisons were significant $(p>0.05)$.

Animals that learned to maintain their leg in a flexed position exhibited fewer responses over time (Fig. 2E). Nonpretrained rats that failed to learn after lidocaine treatment (NoPreTrn $\rightarrow$ Lido) responded at a high rate when tested with response-contingent shock (min 31-6), but this experience did not bring about an increase in flexion duration, our index of learning. An ANOVA confirmed that the main effects of time, pretraining condition, drug treatment, and their interactions were statistically significant (all $F$ values $>8.00, p<0.01$ ). Post hoc comparisons of the group means (Fig. $2 F$ ) showed that the untrained lidocaine-treated group differed from the other three $(p<0.05)$. No other comparison approached significance $(p>0.05)$. Because response duration provides a straightforward measure of learning, and because a failure to learn was routinely accompanied by an increase in the rate of responding in all of the subsequent experiments, we focus our analyses on response duration.

\section{Blocking communication between the spinal cord and periphery interferes with the acquisition, but not the maintenance, of the learned response}

Anesthetizing the lumbosacral spinal cord with lidocaine blocked a spinally mediated reflex (tail withdrawal from radiant heat) and learning but had no effect when applied after the learned response was acquired. The latter observation implies that spinal neurons are not needed to maintain the acquired response over time: that learning induces a peripheral modification that maintains the flexion response. Alternatively, it could be argued that there was limited drug diffusion to the ventral (motor) region of the spinal cord; and for this reason, the essential neural output was not completely blocked. To address this possibility, we targeted the sciatic nerve, which conducts sensory/motor signals to/ from the spinal cord and tibialis anterior muscle. We did so in two ways: by applying lidocaine to the sciatic nerve and by cutting the nerve.

To examine whether lidocaine applied to the sciatic nerve affects the maintenance of the instrumental response, rats had their sciatic nerve exposed before behavioral testing. After animals were prepared for instrumental testing, half (PreTrn) received 30 min of training with response-contingent shock. The remaining animals (NoPreTrn) were set up in the same manner but did not receive training. Twenty-five minutes into the training session, the exposed sciatic nerve was bathed in lidocaine (Lido; $50 \mu \mathrm{l}, 40 \%$ ) or vehicle (Veh). Finally, all animals were tested for $30 \mathrm{~min}$ with response-contingent shock. The full design involved a 2 (PreTrn or NoPreTrn) $\times 2$ (Lido or Veh) factorial $(n=6)$. Data from the training (min 1-30) and test phases (min 31-60) were analyzed separately, with time bin serving as a within-subjects variable.

Before lidocaine was applied to the sciatic nerve, rats exposed to response-contingent shock exhibited a progressive increase in flexion duration indicative of learning $\left(F_{(29,290)}=3.09, p<\right.$ 0.0001 ) (Fig. $3 A$ ). After lidocaine treatment, previously untrained rats (NoPreTrn $\rightarrow$ Lido) failed to learn. Most importantly, lidocaine did not affect performance in rats that had already acquired the learned response (PreTrn $\rightarrow$ Lido). An ANOVA confirmed that drug treatment had a significant effect $\left(F_{(1,20)}=6.75, p=\right.$ $0.0172)$ and that it interacted with pretraining condition $\left(F_{(1,20)}\right.$ $=4.63, p=0.0439)$. The main effect of time and all higher-order interactions with pretraining condition and drug treatment were also statistically significant (all $F$ values $>1.79, p<0.05$ ). Post hoc comparisons of the group means (Fig. $3 B$ ) during the $30 \mathrm{~min}$ test session showed that the group that had no pretraining before lidocaine administration (NoPreTrn $\rightarrow$ Lido) differed from the other three $(p<0.05)$. No other group comparisons were statistically significant $(p>0.05)$.

A similar procedure was used to evaluate the effect of cutting the sciatic nerve. Again, the sciatic nerve was exposed before behavioral testing, and rats were prepared for instrumental testing. Half of the animals (PreTrn) then received $30 \mathrm{~min}$ of training with response-contingent shock while the remaining rats (NoPreTrn) received nothing. Twenty-five minutes into the training session, half of the animals in each condition (trained or untrained) received a complete sciatic transection (Cut) or nothing (NoCut). During the $30 \mathrm{~min}$ of testing, all rats received response-contingent shock. The full design involved a 2 (PreTrn or NoPreTrn $) \times 2$ (Cut or NoCut) factorial $(n=8)$. Data from the training ( $\min 1-30)$ and test phases (min 31-60) were analyzed separately, with time bin serving as a within-subjects variable. 

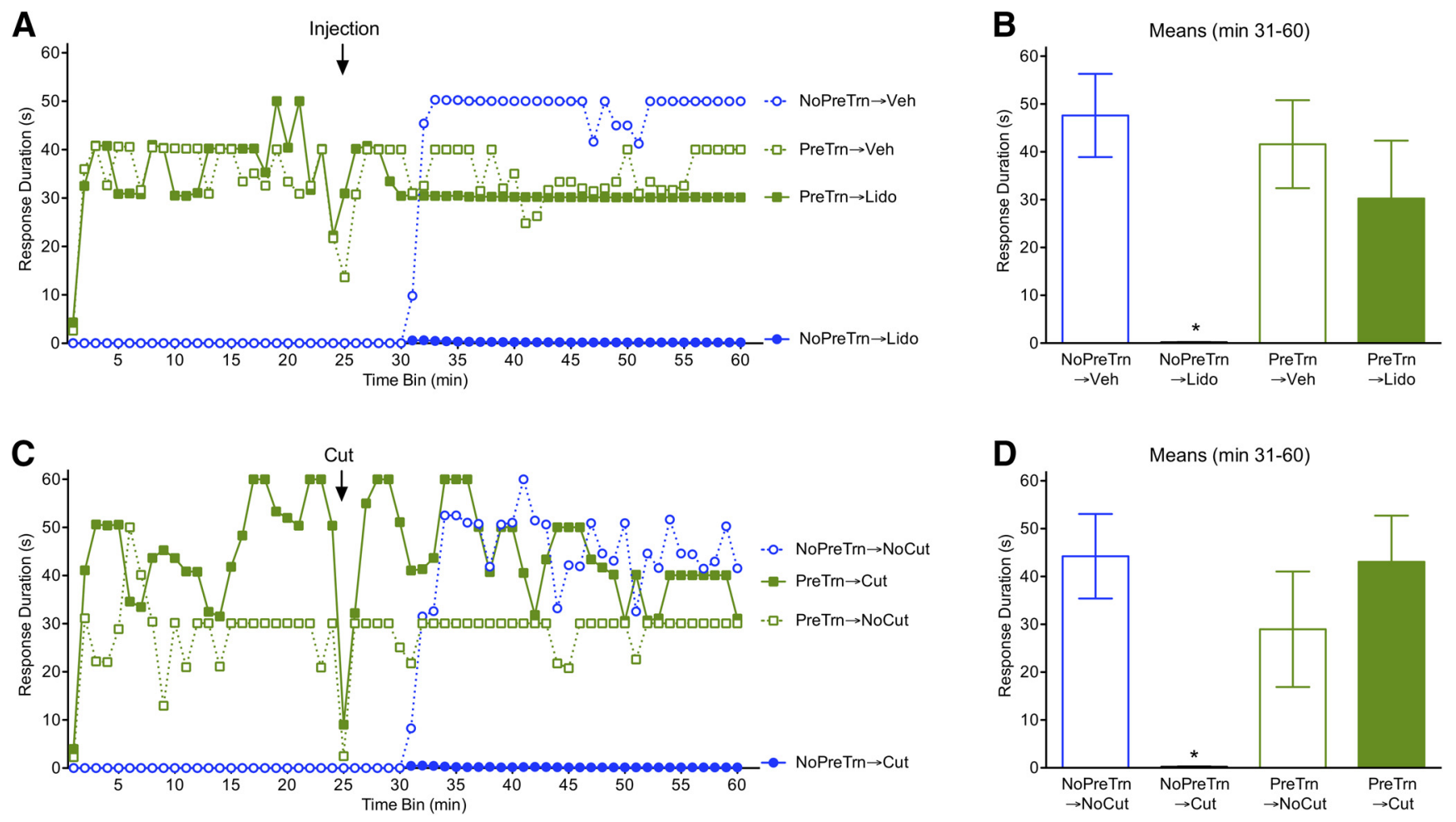

Figure 3. Cutting communication with the spinal cord disrupts acquisition, but not the maintenance, of the learned response. A, Rats received pretraining (PreTrn) or nothing (NoPreTrn), and the drug was infused after $25 \mathrm{~min}(n=6)$. Rats that had not been pretrained and received the vehicle (NoPreTrn $\rightarrow$ Veh) exhibited an increase in flexion duration when tested with response-contingent shock. This learning was blocked by lidocaine applied to the sciatic nerve (NoPreTrn $\rightarrow$ Lido). Lidocaine had no effect on the performance of the learned response in pretrained rats (PreTrn $\rightarrow$ Lido). Lines indicate mean response duration over time during training and testing. B, Mean performance after drug infusion (min 31-60). C, Rats received pretraining (PreTrn) or nothing (NoPreTrn), and the sciatic nerve was cut at $25 \mathrm{~min}(n=8)$. Rats that had not been pretrained and received no cut (NoPreTrn $\rightarrow$ NoCut) exhibited an increase in flexion duration when tested with response-contingent shock. This learning was blocked by a sciatic transection (NoPreTrn $\rightarrow$ Cut). Cutting the sciatic nerve had no effect on the performance of the learned response in pretrained rats (PreTrn $\rightarrow$ Cut). Lines indicate mean response duration over time during training and testing. $\boldsymbol{D}$, Mean test performance (min 31-60). Error bars indicate SE. Asterisks indicate $p<0.05$.

As expected, rats exposed to response-contingent shock exhibited an increase in response duration $\left(F_{(29,290)}=3.83\right.$, $p<0.0001$ ) (Fig. 3C). Cutting the sciatic nerve prevented learning in previously untrained rats but only had a transient effect on performance in pretrained subjects. An ANOVA confirmed that group performance during the $30 \mathrm{~min}$ test session depended on both pretraining condition and whether the sciatic nerve was cut $\left(F_{(1,20)}=12.78, p=0.0019\right)$. There was also a significant effect of time $\left(F_{(29,580)}=1.59, p=0.0263\right)$. No other statistical term was significant (all $F$ values $<3.39, p>0.05$ ). Post hoc comparisons of the group means (Fig. 3D) showed that the animals that were not pretrained before the sciatic cut (NoPreTrn $\rightarrow$ Cut) differed from the other three $(p<0.05)$. No other group comparison was significant $(p>0.05)$.

\section{Excising the spinal cord blocks acquisition, but not the maintenance, of the learned response}

To verify that neurons within the spinal cord play no role in the maintenance of the learned response, we also assessed the effect of removing the spinal cord between L3 and S3 after rats had undergone $25 \mathrm{~min}$ of training. This portion was targeted because prior work has shown that learning is mediated by neurons within this region (Liu et al., 2005). Further, intramuscular application of fluorescent tracers into the tibialis anterior muscle labels motoneurons within the L4-L5 region. One group of rats underwent $30 \mathrm{~min}$ of instrumental training (PreTrn) while another remained unshocked (NoPreTrn) $(n=2)$. Both groups had the spinal cord removed after $25 \mathrm{~min}$ and, 5 min later, were tested with response-contingent shock for $30 \mathrm{~min}$.
Animals trained with response-contingent shock (PreTrn) learned to maintain the shocked leg in a flexed position [mean response duration $( \pm \mathrm{SE})=46.6 \pm 10.2]$, while the untreated animals (NoPreTrn) kept the hind leg in an extended position $(0.0 \pm 0.0)$ An ANOVA confirmed that this difference was statistically significant $\left(F_{(1,2)}=20.97, p=0.0445\right)$. Animals that did not receive pretraining failed to learn when tested with responsecontingent shock [mean response duration $( \pm \mathrm{SE})=0.52 \pm$ 0.31 , while animals in the pretrained condition continued to maintain a flexion response $(60.0 \pm 0.00)$. Animals in the PreTrn condition that maintained a flexion response exhibited a low rate of responding $(0.00 \pm 0.00)$. As observed after a sciatic cut, rats in the NoPreTrn condition that failed to learn responded at a high rate [mean response number ( \pm SE) $192.98 \pm 10.10$ ]. Independent ANOVAs confirmed that these group differences were statistically significant (both $F$ values $>21.99, p<0.0426$ ).

In summary, pharmacologically or surgically disrupting the link between the spinal cord and the site of stimulation prevented learning but had no effect on the maintenance of the behavioral response. These results imply that the outcome of learning (an increase in flexion duration) is preserved, in part, by a peripheral modification.

\section{Spinal neurons mediate the metaplastic effects of instrumental training}

Interestingly, exposure to response-contingent shock has been shown to impact future learning potential (Crown et al., 2002a; Grau et al., 2012). For example, after animals are trained with the 

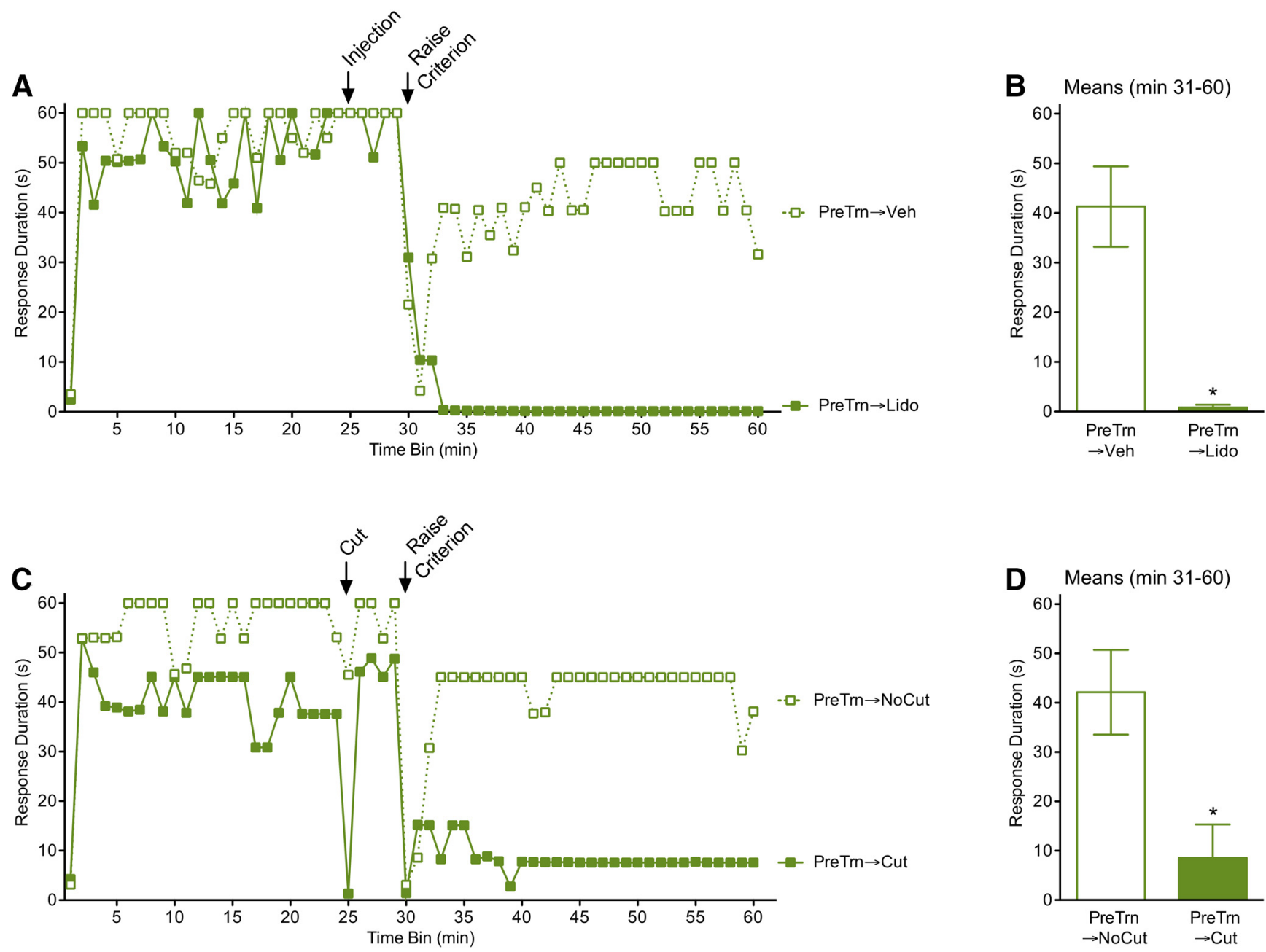

Figure 4. Cutting communication with the spinal cord prevents learning when animals are tested with a higher response criterion. $\boldsymbol{A}$, Rats were pretrained (PreTrn), and the drug was infused onto the sciatic nerve after $25 \mathrm{~min}(n=6)$. The response criterion was then increased by $4 \mathrm{~mm}$ by adding additional fluid to the underlying salt solution. Pretrained rats that received the vehicle (PreTrn $\rightarrow$ Veh) were able to learn when tested with a higher response criterion. Administration of lidocaine (PreTrn $\rightarrow$ Lido) blocked this effect. Lines indicate mean response duration over time during training and testing. B, Mean performance when tested with a high response criterion (min 31-60). C, Rats were pretrained (PreTrn), and the sciatic nerve was cut at min 25. The response criterion was then increased by $4 \mathrm{~mm}(n=8)$. Animals were able to learn whether the sciatic nerve was intact (PreTrn $\rightarrow$ NoCut). Cutting the nerve prevented learning $($ PreTrn $\rightarrow$ Cut). Lines indicate mean response duration over time during training and testing. D, Mean performance when tested with a high response criterion (min 31-60). Error bars indicate SE. Asterisks indicate $p<0.05$.

usual response criterion, they can learn when tested with a higher response criterion that requires a stronger flexion response. Minus pretraining, animals fail to learn when tested with a higher criterion. These observations imply that training enables subsequent learning, a kind of plasticity of plasticity (metaplasticity; Fig. 1B) (Abraham and Bear, 1996; Abraham, 2008; Ferguson et al., 2008; Grau et al., 2014; Grau and Huang, 2018). Does this process also depend on a peripheral modification? To evaluate this possibility, pretrained rats received either intrathecal lidocaine or had the sciatic nerve cut and were tested with a higher response criterion.

To test the effect of lidocaine treatment, spinally transected rats had intrathecal catheters implanted at the time of surgery. The next day, they were set up for instrumental training, and the underlying solution was adjusted so that the contact electrode was submerged by $4 \mathrm{~mm}$ (normal response criterion). After 25 min of training, half the animals (PreTrn $\rightarrow$ Lido) received a $20 \mu \mathrm{l}$ intrathecal injection of lidocaine (40\%). The remaining rats (PreTrn $\rightarrow$ Veh) were given vehicle. Five minutes later, the response criterion was raised to $8 \mathrm{~mm}$ (high response criterion), by adding a fixed volume of solution, and animals were tested for another $30 \mathrm{~min}$. The design involved two groups (Lido or Veh) with 6 animals per condition. Data from the training (min 1-30) and test phases (min 31-60) were analyzed separately, with time bin serving as a within-subjects variable.

As expected, the pretrained groups exhibited an increase in response duration $\left(F_{(29,290)}=8.56, p<0.0001\right)$ and did not differ before drug treatment (all $F$ values $<1.0, p>0.05$ ). Pretrained rats that received vehicle (PreTrn $\rightarrow$ Veh) were able to learn when tested with a higher response criterion (Fig. $4 A, B$ ). Rats treated with lidocaine (PreTrn $\rightarrow$ Lido) failed to learn with the higher criterion. An ANOVA confirmed that drug treatment had a significant effect $\left(F_{(1,10)}=20.50, p=0.0011\right)$ and that the change in response duration observed over time depended on drug treatment $\left(F_{(29,290)}=2.24, p=0.0004\right)$. The main effect of time was not significant $\left(F_{(29,290)}=1.07, p>0.05\right)$.

An analogous design was used to test the effect of cutting the sciatic nerve. A day after rats received a spinal transection, the sciatic nerve was exposed, and rats were set up for training with the contact electrode submerged by $4 \mathrm{~mm}$. After $25 \mathrm{~min}$ of 
training, half the animals (PreTrn $\rightarrow$ Cut) had the sciatic nerve cut as described above. Five minutes later, the response criterion was raised to $8 \mathrm{~mm}$, and the capacity to learn was assessed over the next $30 \mathrm{~min}$. The design involved two groups (Cut or NoCut), with 8 animals per condition. Data from the training (min 1-30) and test phases (min 31-60) were analyzed separately, with time bin serving as a within-subjects variable.

During the $30 \mathrm{~min}$ of pretraining, both groups exhibited an increase in flexion duration across time $\left(F_{(29,406)}=10.16\right.$, $p<0.0001)$. Pretrained rats that did not receive a sciatic transection (PreTrn $\rightarrow$ NoCut) were able to learn when tested with a higher response criterion. Cutting the sciatic nerve (PreTrn $\rightarrow$ Cut) blocked this learning (Fig. 4C,D). An ANOVA yielded a significant main effect of sciatic transection $\left(F_{(1,14)}=8.22, p=\right.$ $0.0124)$. The main effect of time and its interaction with surgery condition were also significant (both $F$ values $>2.05, p<0.005$ ).

In summary, training with response-contingent shock enabled learning when animals were tested with a higher response criterion. Inhibiting neural activity with lidocaine, or cutting the sciatic nerve, blocked the capacity to learn. The findings imply that this example of learning to learn (behavioral metaplasticity) (Schmidt et al., 2013) depends on spinal neurons. Conversely, the findings reported earlier showed that these same manipulations had no effect on the maintenance of the behavioral response. Together, it appears that the CNS is necessary for new learning, but a peripheral modification is sufficient to maintain the trained response (an increase in flexion duration).

\section{Tonic EMG muscle activity is modulated by instrumental learning}

Our results suggest that instrumental learning brings about a peripheral modification that contributes to the maintenance of a flexion response. We assumed that training with response-contingent stimulation increases flexion duration because it enhances the electrical response evoked within the tibialis anterior muscle. Alternatively, training might simply reset the resting state of the muscle, inducing a kind of "latch state" that maintains the leg in a flexed position. To evaluate these alternatives, we used EMG to assess tonic EMG activity within the tibialis anterior muscle during, and after, training with controllable or uncontrollable stimulation. To determine whether the effects observed reflect a peripheral modification, additional animals were tested after a sciatic transection (Cut).

Rats were set up for instrumental training and then received three AC shocks ( $150 \mathrm{~ms} ; 150 \mathrm{~ms}$ interstimulus interval; $0.5 \mathrm{~mA}$ ) to the tibialis anterior muscle spaced $20 \mathrm{~s}$ apart. Over the next minute, the underlying salt solution was adjusted to submerge the contact electrode tip by $4 \mathrm{~mm}$, and EMG activity was record. Animals then received $30 \mathrm{~min}$ of training with response-contingent shock (Master) or noncontingent shock (Yoked) $(n=6)$. EMG activity was recorded during training, minus the periods when AC stimulation was being applied. Immediately following training, a burst of three shocks was applied, and EMG activity was recorded for $1 \mathrm{~min}$. A second test was performed $30 \mathrm{~min}$ later. Two additional groups $(n=6)$ of rats were set up and tested as described above but received a sciatic transection (Cut) after 25 min of training.

As expected, animals exposed to controllable (Master), but not uncontrollable (Yoked), shock exhibited an increase in flexion duration during training (Fig. 5A). An ANOVA confirmed that the main effects of training condition, time, and their interaction were all statistically significant (all $F$ values $>3.65$, $p<0.0001)$. During this period, rats in the Master condition exhibited greater EMG activity within the muscle, relative to rats in the Yoked condition (Fig. 5B). An ANOVA confirmed that the main effects of training condition and time were statistically significant (both $F$ values $>12.16, p<0.0001$ ). The magnitude of this group difference did not vary across time $\left(F_{(29,290)}<1.0\right.$, $p>0.05)$. Likewise, the EMG recorded over a $1 \mathrm{~min}$ period following a burst of muscle stimulation applied immediately $(0 \mathrm{~min})$ or $30 \mathrm{~min}$ after training (Fig. $5 C$ ) revealed a main effect of training condition $\left(F_{(1,10)}=7.24, p=0.0227\right)$. Neither the main effect of time, nor its interaction with training condition, approached statistical significance (both $F$ values $\langle 1.0, p>$ $0.05)$.

Two additional groups were set up and trained in the same manner but received a sciatic cut after $25 \mathrm{~min}$ of training. Before the sciatic cut, animals that received controllable shock (Master) learned to maintain the stimulated leg in a flexed position, relative to rats in the Yoked condition (Fig. 5D). An ANOVA showed that the groups differed $\left(F_{(1,6)}=16.61, p=0.0065\right)$ and that the magnitude of this difference varied with time $\left(F_{(24,144)}=\right.$ $1.72, p=0.0273)$. The overall group difference in response duration survived a sciatic cut at minute $25\left(F_{(1,6)}=3528.31, p<\right.$ $0.0001)$. As reported above, the transection induced a transient disruption in behavioral performance, yielding a main effect of time and a time $\times$ training condition interaction that approached statistical significance (both $F$ values $>2.52, p<0.0675$ ).

Animals given controllable stimulation exhibited greater EMG activity during training (Fig. $5 E$ ). The magnitude of this effect appeared greater at the start of training. An ANOVA confirmed that the main effects of time and training condition, as well as their interaction, were statistically significant (all $F$ values $>2.45, p<0.0328)$. Cutting the sciatic nerve produced a burst of electrical activity within the muscle and revealed a robust effect of training condition that faded within a few minutes. An ANOVA confirmed that animals in the Master condition exhibited greater EMG activity after the sciatic cut and that the magnitude of this effect varied with time (both $F$ values $>2.62$, $p<0.05)$. Immediately after training ended $(0 \mathrm{~min})$, no effect of training was observed after a burst of electrical stimulation (Fig. $5 F$ ). However, a retest at $30 \mathrm{~min}$ revealed a lasting effect of training. An ANOVA confirmed that there was a significant effect of training condition that varied with time $\left(F_{(1,10)}=5.72, p=\right.$ 0.0379 ). No other term was statistically significant (all $F$ values $<$ $1.26, p>0.05)$

Close inspection of the learning (Fig. $5 A, D$ ) and EMG (Fig. $5 B, E)$ data revealed an unexpected result: instituting a responseoutcome relation impacted both response duration and EMG activity within the first minute of training (Fig. 5G,H). Independent ANOVAs confirmed that these group differences were statistically significant (both $F$ values $>4.95, p<0.0378$ ). The fact an instrumental relation has an immediate effect on performance suggests that a biologically prepared circuit gates the motoneuron output in relation to limb position (Grau et al., 2012).

In summary, we found that animals given response-contingent stimulation exhibit greater EMG activity, and this effect survived a sciatic cut. We also observed that cutting the sciatic nerve led to a surge in EMG activity. This is consistent with prior work demonstrating that nerve injury can trigger a barrage of neural activity (Chi et al., 1993; Costigan et al., 2009). Importantly, an effect of behavioral control was still evident immediately after the sciatic cut. Over the subsequent $5 \mathrm{~min}$, EMG activity may wane as presynaptic stores are depleted. From this perspective, 

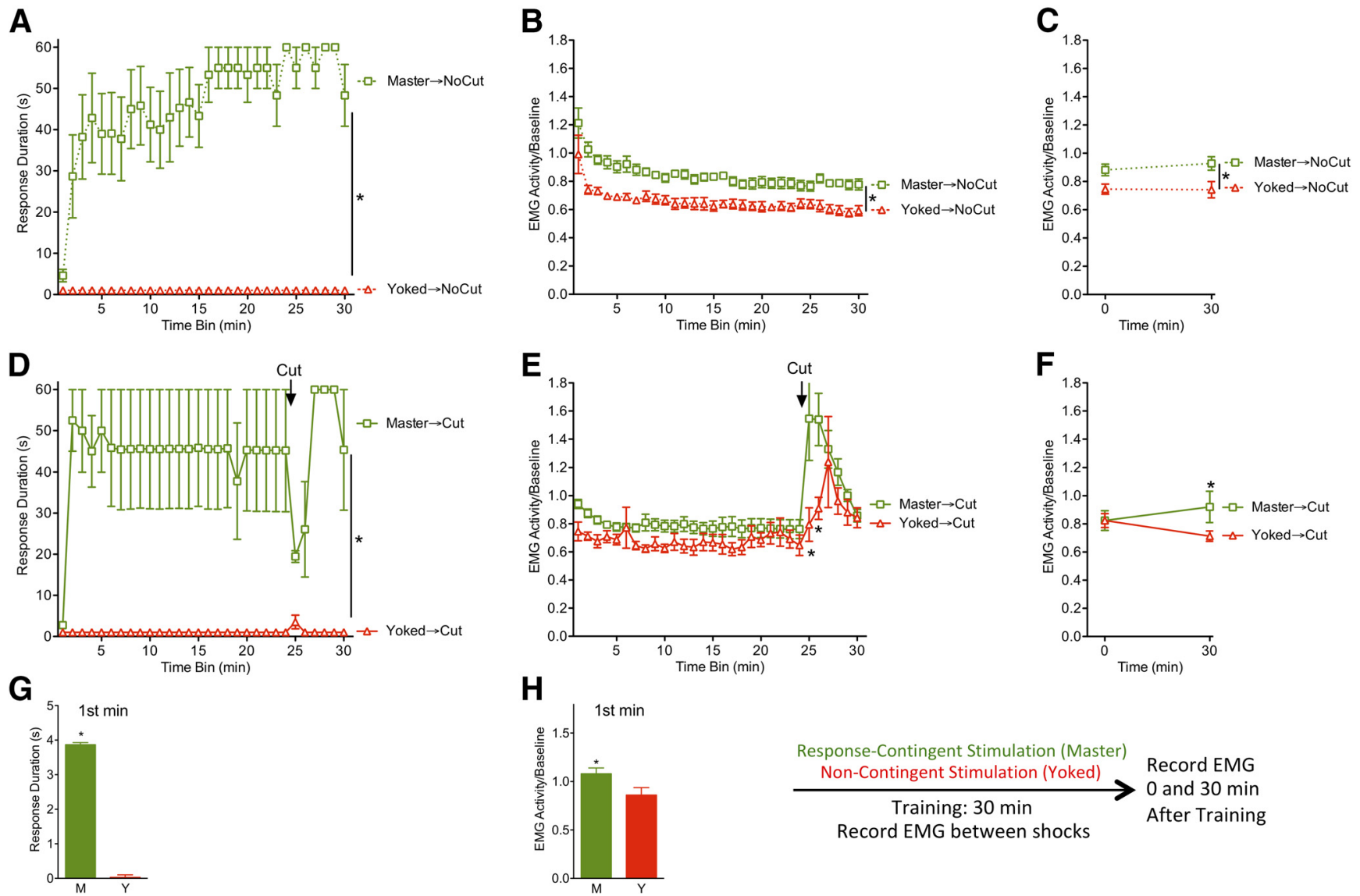

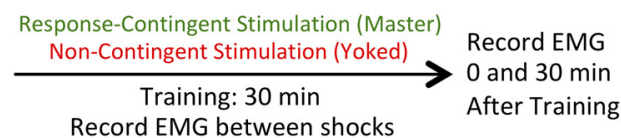

Record EMG between shocks

Figure 5. Instituting an instrumental relation impacts tonic EMG activity within the muscle. $\boldsymbol{A}$, Spinally transected rats that received 30 min of training with response-contingent shock (Master) exhibited a progressive increase in flexion duration $(n=6)$. This learning was not observed in animals that received shock independent of leg position (noncontingent; Yoked). Lines indicate mean response duration over time during training. $\boldsymbol{B}$, Tonic EMG activity within the tibialis anterior muscle during training between periods of muscle stimulation. Less activity was observed in rats that received uncontrollable stimulation (Yoked). C, EMG activity at 0 and 30 min after training over a 1 min period immediately after the presentation of 3 shocks to the muscle. Instrumental training had an effect that lasted $30 \mathrm{~min}$. D, Spinally transected animals received response-contingent shock (Master) or noncontingent shock (Yoked), followed by sciatic transection (Cut) ( $n=6$ ). Master animals exhibited an increase in response duration that survived a sciatic cut. Lines indicate mean response duration over time during training. $\boldsymbol{E}$, Tonic EMG activity within the tibialis anterior muscle during training between periods of muscle stimulation. Animals that received uncontrollable stimulation (Yoked) generally exhibited lower EMG activity, and this effect was still evident immediately after the sciatic cut. In addition, we found that cutting the sciatic nerve produced a transient increase in EMG activity. $\boldsymbol{F}$, EMG activity at 0 and 30 min after training over a 1 min period immediately after the presentation of 3 shocks to the muscle. At the end of training ( 0 min), shortly after the sciatic cut, there was no effect of training. A significant effect of training condition was observed 30 min later. G, Collapsed across the NoCut and Cut conditions, animals trained with response-contingent shock (Master; M) exhibited an increase in flexion duration relative to those given noncontingent shock (Yoked; $Y$ ) during the first minute of training. $\boldsymbol{H}$, Collapsed across the NoCut and Cut conditions, animals trained with response-contingent shock (Master; M) exhibited higher EMG activity relative to those given noncontingent shock (Yoked; Y) during the first minute of training. Error bars indicate SE. Asterisks indicate $p<0.05$.

once neural activity subsides and stores are replenished, a Master-Yoke effect can be resolved (30 min after training).

Instrumental training strengthens the evoked EMG, and this effect survives a sciatic transection

The EMG data reported above were limited by our inability to record activity during the period of muscle stimulation (because the applied voltage obscures the EMG signal). For this reason, we cannot tell from those data whether training impacts the electrical response evoked by the efferent motoneurons. To address this issue, we assessed the EMG response elicited by sciatic nerve stimulation in animals that had previously received controllable (Master) or uncontrollable (Yoked) shock. In addition, an unshocked control was included to assess the effect of shock exposure per se As in the prior experiment, additional animals were tested after the sciatic nerve was transected.

A day after rats received a spinal cord transection, the sciatic nerve was exposed and a nerve cuff was positioned on the sciatic nerve. Animals were then placed in the training apparatus and had electrodes implanted into the tibialis anterior muscle. Baseline EMG responses were assessed by stimulating (1V) the sciatic nerve 3 times. The EMG recording equipment was then turned off, and AC stimulation was applied to set flexion force and contact electrode depth. One-third of the rats (Master) received $30 \mathrm{~min}$ of training with response-contingent shock. Another third (Yoked) received the same distribution of shock independent of leg position. The remaining animals served as the unshocked controls. At the end of training, half the animals in each training condition had the sciatic nerve transected. The evoked EMG response was then reassessed in all animals. The full design involved a 3 (Master, Yoked, or Unshocked) $\times 2$ (Cut or NoCut) factorial $(n=6)$. An ANOVA was used to analyze the training data with time bin (1-30) serving as a within-subjects variable. The $100 \mathrm{~ms}$ EMG recordings were broken into $10 \mathrm{~ms}$ time bins and the average response computed. The mean response amplitude observed within each time bin was then included as a within-subjects variable. To control for variation in EMG amplitude across subjects, an ANCOVA was used to 


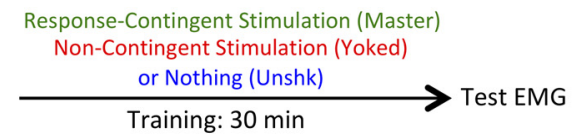

A

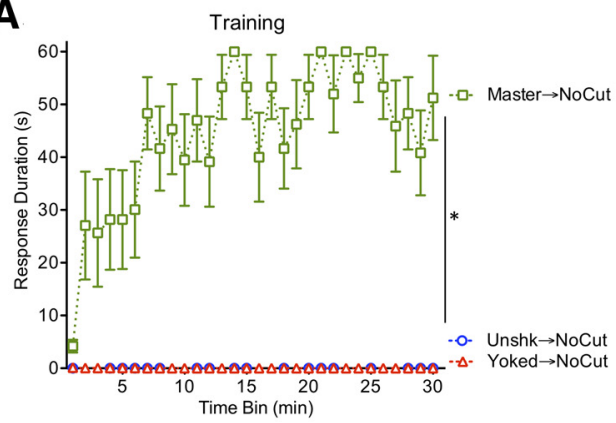

D

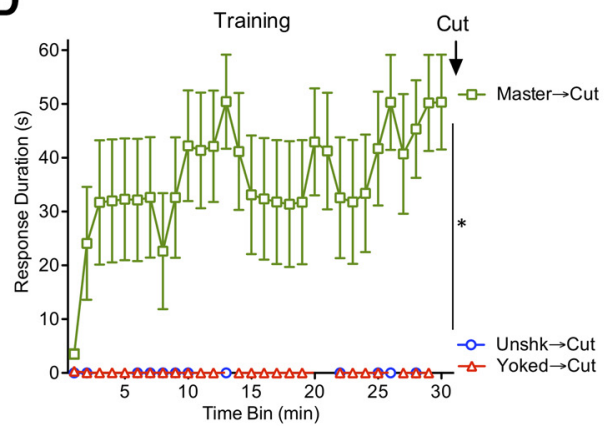

B

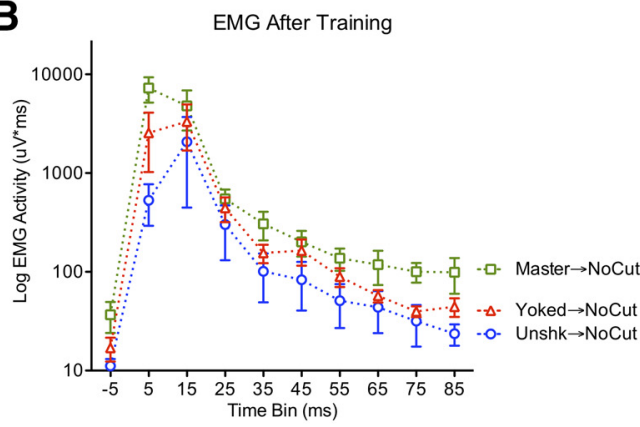

E

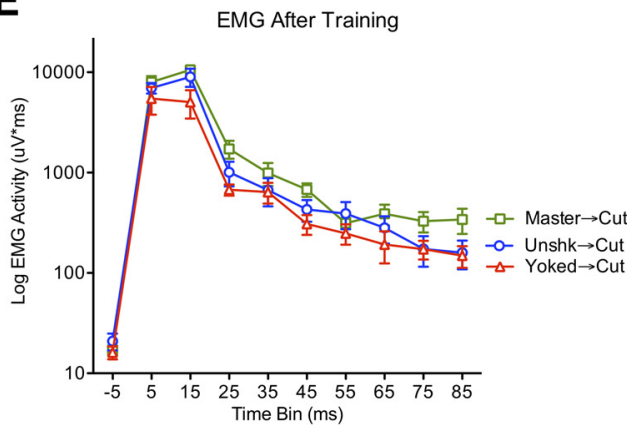

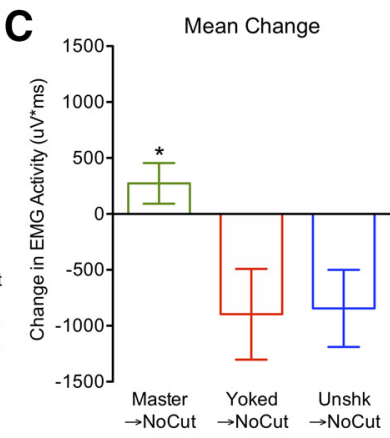

$\mathbf{F}$

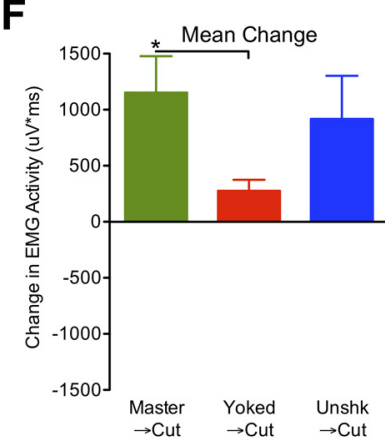

Figure 6. Instrumental training augments the EMG response elicited by sciatic stimulation after communication with the spinal cord has been cut. $\boldsymbol{A}$, Spinally transected rats that received 30 min of training with response-contingent shock (Master) exhibited a progressive increase in flexion duration $(n=6)$. This learning was not observed in animals that received shock independent of leg position (noncontingent; Yoked). Lines indicate mean response duration over time during training. $\boldsymbol{B}$, The rectified EMG response (log values) to stimulation of the sciatic nerve, collapsed across $10 \mathrm{~ms}$ bins. Master animals exhibited greater EMG activity. $\boldsymbol{C}$, The mean change in EMG activity, collapsed across the test period. D, Spinally transected animals received response-contingent shock (Master), noncontingent shock (Yoked), or nothing (Unshk), followed by sciatic transection (Cut) ( $n=6)$. Cutting the sciatic nerve generally augmented the EMG response. Lines indicate mean response duration over time during training. $\boldsymbol{E}$, Master animals exhibited a stronger EMG relative to Yoked animals, and this difference survived a sciatic cut. $\boldsymbol{F}$, The mean change in EMG activity during testing. Error bars indicate SE. Asterisks indicate $p<0.05$.

analyze the post-training data, with pretraining EMG activity serving as the covariate.

Before training, the EMG response elicited by stimulation of the sciatic nerve did not differ across groups (all $F$ values $<1.0$, $p>0.05$ ). As expected, exposure to controllable shock (Master), but not uncontrollable shock (Yoked), elicited an increase in flexion duration across the $30 \mathrm{~min}$ of training (Fig. 6A,D). An ANOVA confirmed that the main effects of training condition, time, and their interaction were all statistically significant (all $F$ values $>3.61, p<0.0001)$.

At the end of training, Master rats that had an intact sciatic nerve (Master $\rightarrow$ NoCut) exhibited a larger EMG response relative to both the Yoked $\rightarrow$ NoCut and Unshk $\rightarrow$ NoCut groups (Fig. 6B,C). Nerve injury per se enhanced the evoked response. (Fig. 6E,F). Most importantly, cutting the sciatic nerve did not eliminate the effect of controllable stimulation. An ANCOVA, using the pretraining EMG activity as a covariate, confirmed that the main effect of training condition and sciatic transection were statistically significant (both $F$ values $>8.37, p<0.0013$ ). Importantly, the effect of training did not depend on whether the sciatic nerve was intact or cut $\left(F_{(2,29)}=1.78, p>0.05\right)$ (Fig. $6 E$, $F)$. As expected, the amplitude of the response elicited varied with time $\left(F_{(9,261)}=10.27, p<0.0001\right)$, and the magnitude of this effect depended on training history $\left(F_{(18,261)}=3.44, p<\right.$ 0.0001). The extent to which a sciatic cut amplified the EMG response varied with time $\left(F_{(9,261)}=11.77, p<0.0001\right)$, but the magnitude of this effect did not depend on training history $\left(F_{(18,261)}=1.58, p>0.05\right)$. Post hoc comparisons of the overall group means showed that animals in the Master condition differed from both the Yoked and unshocked groups $(p<0.05)$. No other comparisons were statistically significant $(p>0.05)$. Additional analyses, which compared the Master and Yoked animals within each surgical condition, confirmed that these groups differed in both the NoCut $\left(F_{(1,10)}=5.66, p=0.0386\right)$ and Cut $\left(F_{(1,10)}=5.51, p=0.0409\right)$ conditions.

The results show that training with response-contingent shock (Master) amplifies the evoked EMG response to sciatic stimulation. Importantly, this effect was not observed in animals that received the same amount of shock independent of leg position (Yoked). We again observed that cutting the sciatic nerve increases EMG activity within the muscle. Because nerve injuryinduced neural activity would activate muscles in a nonselective manner, it would not produce a selective behavioral modification (increased flexion) akin to learning. More importantly, the Master-Yoke difference in the evoked EMG response was still evident after a sciatic transection.

Intramuscular curare blocks acquisition and maintenance of the instrumental response

We have established that training induces a peripheral modification that augments the EMG response elicited by the motor output and that this effect survives a sciatic transection. This implies 
A
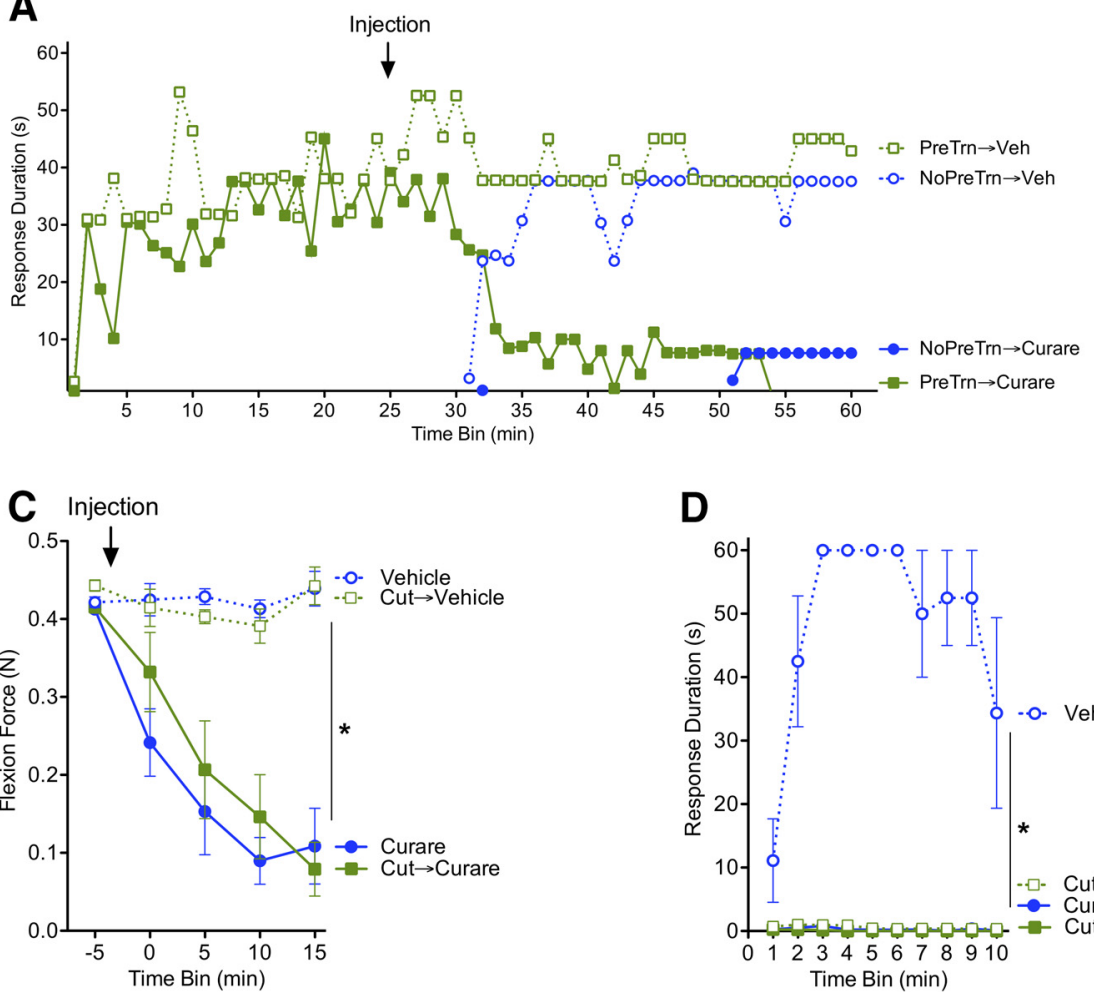

D

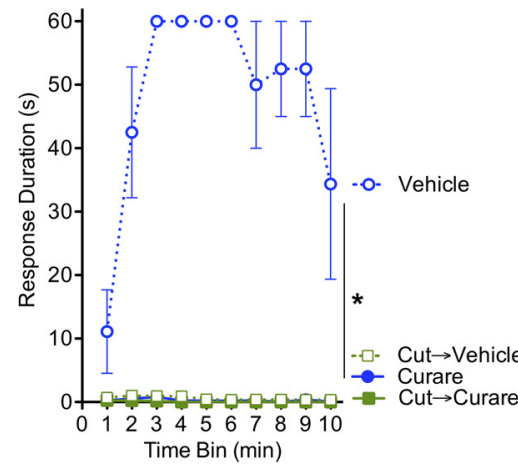

B

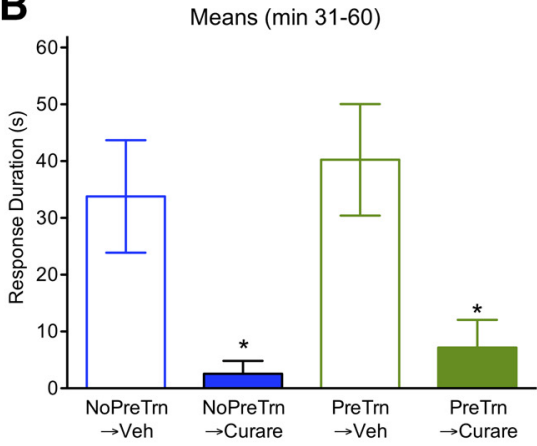

E

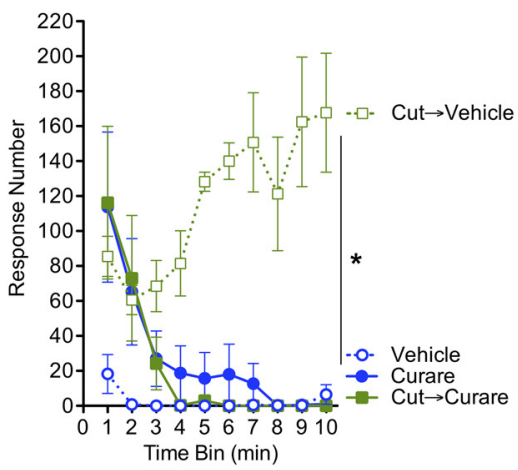

Figure 7. Intramuscular application of curare blocks the acquisition and maintenance of the learned response. $A$, Spinally transected rats received training with response-contingent shock (PreTrn), or nothing (NoPreTrn), and an intramuscular (intramuscular) injection was given at $25 \mathrm{~min}(n=8)$. Rats that were not pretrained and received the vehicle (NoPreTrn $\rightarrow$ Veh) learned to maintain the hindlimb in a flexed position. This learning was blocked by curare (NoPreTrn $\rightarrow$ Curare). Curare also caused the instrumental response to wane in previously trained animals (PreTrn $\rightarrow$ Curare). Lines indicate mean response duration over time during training and testing. B, Mean performance during the testing period (min 31-60). C, Animals that received curare exhibited a reduction in flexion force relative to vehicle-treated controls, and this effect survived a sciatic transection (Cut) $(n=4)$. D, Vehicle-treated rats given response-contingent shock exhibited an increase in response duration, and this learning was blocked by pretreatment with curare or a sciatic transection (Cut). $E$, Vehicle-treated rats that learned to maintain their leg in a flexed position exhibited fewer responses. Vehicle-treated rats that received a sciatic transection (Cut + Vehicle) failed to learn but continued to respond at a high rate. Animals pretreated with curare responded during the first few minutes of testing, but this effect waned within a few minutes. Error bars indicate SE. Asterisks indicate $p<0.05$.

that the change in the behavioral response involves an alteration at the NMJ. In adult animals, neurochemical transmission at the NMJ depends on the release of the neurotransmitter ACh, which activates nAChRs, producing an endplate response by opening monovalent cation channels permeable to both $\mathrm{Na}^{+}$and $\mathrm{K}^{+}$ (Sanes and Lichtman, 2001). This response, in turn, results in the opening of voltage-gated channels to generate an action potential in the muscle fiber. If the training-induced increase in flexion duration depends on neuronal drive (ACh release and the engagement of $\mathrm{ACh}$ receptors), disrupting neurochemical transmission at the AChR should eliminate the effect. The present experiment addresses this issue by testing whether the local (intramuscular [i.m.]) application of the AChR antagonist curare disrupts the maintenance and performance of the learned response.

We first verified that intramuscular application of curare affects motor learning. Spinally transected rats were set up for instrumental training as described above. Curare $(240 \mu \mathrm{g})$ or its vehicle was then injected into the tibialis anterior muscle. Five minutes later, rats underwent $30 \mathrm{~min}$ of training. The design involved two groups (Curare vs Veh) with 6 animals per condition. The training data were analyzed across time (min 1-30), which served as a within-subjects variable.

During setup, we recorded the shock intensity required to elicit a flexion force of $0.4 \mathrm{~N}$ before and after drug administration. Administration of the vehicle had no effect [mean change in shock intensity $( \pm \mathrm{SE})=0.00 \pm 0.00 \mathrm{~mA}]$, but stronger shocks were needed $(0.15 \pm 0.04 \mathrm{~mA})$ after curare $\left(F_{(1,10)}=12.27\right.$, $p=0.0057)$. With initial flexion force equated across groups, there was no difference in the duration of the first shock-elicited flexion response $\left(F_{(1,10)}<1.0, p>0.05\right)$. Over the subsequent $30 \mathrm{~min}$ of testing, vehicle-treated animals exhibited an increase in flexion duration [mean $( \pm \mathrm{SE})=28.67 \pm 2.22$ ], whereas $\mathrm{cu}$ rare-treated rats did not $(0.08 \pm 0.04)\left(F_{(1,10)}=5.57, p=0.040\right)$. As expected, the effect of curare on baseline reactivity shows that electrical stimulation of the muscle elicits some ACh release. More importantly, the blockade of learning implies that the training-induced increase in flexion duration depends on the activation of the AChR.

Next, we assessed the effect of intramuscular curare on the maintenance of the learned response. A day after spinal transection, animals were placed in the test apparatus and were set up for stimulation of the tibialis anterior muscle as described above. Half the animals then received 30 min of training with controllable shock (PreTrn). The remaining animals served as the no pretraining (NoPreTrn) controls. After $25 \mathrm{~min}$, half of the rats in each condition received an intramuscular injection of curare $(240 \mu \mathrm{g})$. The remaining rats were treated with the vehicle. Five minutes later, all of the animals underwent $30 \mathrm{~min}$ of testing with response-contingent shock. The full design involved a 2 $($ PreTrn or NoPreTrn $) \times 2$ (Curare or Veh) factorial $(n=8)$. Data from the training (min 1-30) and test phases (min 31-60) were analyzed separately, with time bin serving as a within-subjects variable. 
Rats that were pretrained with response-contingent shock exhibited an increase in flexion duration $\left(F_{(29,406)}=2.45, p=\right.$ 0.0001 ) and did not differ before drug infusion (all $F$ values $<$ 1.0, $p>0.05$ ) (Fig. 7). Administration of curare blocked learning in rats that had not been pretrained (NoPreTrn $\rightarrow$ Curare). In pretrained animals (PreTrn $\rightarrow$ Curare), curare caused the learned response to wane. An ANOVA performed on the data collected over the $30 \mathrm{~min}$ test session revealed a significant main effect of drug treatment $\left(F_{(1,28)}=19.15, p=0.0002\right)$, a time $\times$ drug interaction $\left(F_{(29,812)}=2.21, p=0.0003\right)$, and a time $\times$ training interaction $\left(F_{(29,812)}=3.07, p=0.0001\right)$. No other effects were significant (all $F$ values $<1.33, p>0.05$ ). Post hoc comparisons of the group means during testing (Fig. $7 B$ ) showed that the curare-treated groups were significantly different from the salinetreated controls $(p<0.05)$. No other comparison was significant $(p>0.05)$.

Because electrical stimulation was applied to the muscle, we anticipated that curare-treated animals would exhibit a behavioral (flexion) response. As predicted, after drug treatment (time bins 31-60), rats given curare exhibited an average response rate (mean $=50.9 \pm 21.7$ per min) only slightly less than that exhibited by the vehicle-treated controls (mean $=68.1 \pm 25.3$ per $\mathrm{min})$. This difference was not statistically significant $\left(F_{(1,28)}=\right.$ 2.40, $p=0.13$ ).

In developing our instrumental learning paradigm, we adopted a methodology wherein initial flexion force was equated before testing. This served two purposes. First, it reduced variability across animals. Second, it negated an alternative interpretation of some results that attributed a failure to learn to a disruption in the capacity to perform the target response. The procedure did, however, introduce some complexities with regard to the present experiment because more intense shock was needed to elicit a comparable flexion force $(0.4 \mathrm{~N})$ in curaretreated animals. The fact curare impaired performance suggests that electrical stimulus drives a flexion response, in part, by triggering the release of $\mathrm{ACh}$ from the motoneurons that innervate the muscle. Because our stimulating electrodes were positioned across the midline of the muscle, which contains the densest region of motoneuron innervation, this was expected. Likewise, it is not surprising that more intense electrical stimulation can elicit a muscular response, because this should directly drive the depolarization-induced $\mathrm{Ca}^{2+}$ release (DICR) that initiates a muscle contraction. What is not clear is whether the moderate shock typically used to elicit a $0.4 \mathrm{~N}$ flexion response is sufficient to activate muscle fibers in the absence of ACh release. Because muscle fibers are much larger than the motor neuron axons/terminals, the size principle suggests that $\mathrm{ACh}$ release will play a predominant role. To explore this issue, rats were set up for instrumental testing and shock intensity was adjusted to elicit a $0.4 \mathrm{~N}$ response. We then applied curare, or its vehicle, intramuscular and assessed flexion force at $5 \mathrm{~min}$ intervals over a period of $15 \mathrm{~min}(n=4)$. To verify drug treatment affects the capacity to learn, animals were then tested for $10 \mathrm{~min}$ with response-contingent shock. Finally, to assess the contribution of efferent output from the spinal cord, two additional groups $(n=4)$ were tested in the same manner after a sciatic transection (Cut).

Curare weakened flexion force over the $15 \mathrm{~min}$ of testing, and the emergence of this effect was unaffected by a sciatic transection (Cut) (Fig. 7C). An ANOVA confirmed that there was a main effect of drug treatment, time, and a drug $\times$ time interaction (all $F$ values $>17.99, p<0.0001$ ). No other term reached statistical significance (all $F$ values $<2.31, p>0.05$ ).
Vehicle-treated rats given response-contingent shock exhibited an increase in flexion duration over the $10 \mathrm{~min}$ of testing (Fig. 7D). Cutting the sciatic nerve, or pretreatment with curare, blocked learning. An ANOVA confirmed that the main effects of drug treatment, sciatic cut, and time, as well as all interactions, were statistically significant (all $F$ values $>5.39, p<0.0001$ ). Post hoc comparisons of the group means confirmed that the vehicletreated group that had not undergone a sciatic transection differed from the other three $(p<0.05)$. No other group comparison was significant $(p>0.05)$.

Vehicle-treated rats that learned to maintain a flexion response exhibited few responses during testing (Fig. 7E). The failure to learn in vehicle-treated rats that had undergone a sciatic transection (Cut $\rightarrow$ Vehicle) was accompanied by a high and sustained rate of responding. Rats pretreated with curare responded at the start of testing, but performance faded within the first few minutes. An ANOVA confirmed that the effects of drug treatment, sciatic cut, and time, as well as all interactions were statistically significant (all $F$ values $>3.87, p<0.0003$ ). The significant three-way interaction indicates that the change in response number over time depends on both drug treatment and whether the sciatic nerve is intact. Post hoc comparisons of the group means confirmed that the sciatic cut group that received vehicle differed from the other three $(p<0.05)$. No other group comparison was significant $(p>0.05)$.

Our results imply that, at the shock intensity normally used to elicit a motor response, performance is largely driven by activation of the efferent motoneurons and the release of $\mathrm{ACh}$. Importantly, this remains true after the communication with the spinal cord is removed by means of a sciatic transection. The fact that electrical stimulation of the muscle can elicit some response after curare treatment suggests that it directly activates muscle fibers (DICR). However, this effect wanes within a few minutes, implying that the sustained responding observed after other treatments that disrupt learning is attributable to stimulationinduced ACh release. Conversely, engaging $\mathrm{Ca}^{2+}$ release (DICR) in the absence of AChR activity appears to bring about a rapid diminution of the response that could contribute to homeostasis.

We earlier noted that training could induce a modification within the muscle that sustains a prolonged contraction (a kind of latch state). The fact that training enhances the evoked electrical response suggests an active process is involved. Likewise, the finding that curare eliminates the learned response implies continued ACh release plays a pivotal role. Nonetheless, it should be noted that curare did not immediately eliminate the learned response, but instead exerted an effect that caused the prolonged flexion to slowly wane over the subsequent 5-7 min. This transient component could reflect a contractile process that contributes to the maintenance of the behavioral response.

\section{Training increases the density of AChR binding}

Using quantitative fluorescence imaging, Akaaboune et al. (1999) showed that muscle activity regulates AChR density at the NMJ. This was quantified using fluorescently labeled $\alpha$-bungarotoxin, which binds to the AChR. We used this technique to explore whether training enhances NMJ function by increasing AChR density. An internal control was obtained by comparing AChR labeling within the trained tibialis anterior muscle to labeling in the untrained (contralateral) muscle.

A day after rats received a spinal transection, they were set up for instrumental training as described above. Animals then received $30 \mathrm{~min}$ of training with response-contingent shock. At 
Collect muscle from

Pretrained (PreTrn) and

Untrained (NoPreTrn) leg
A

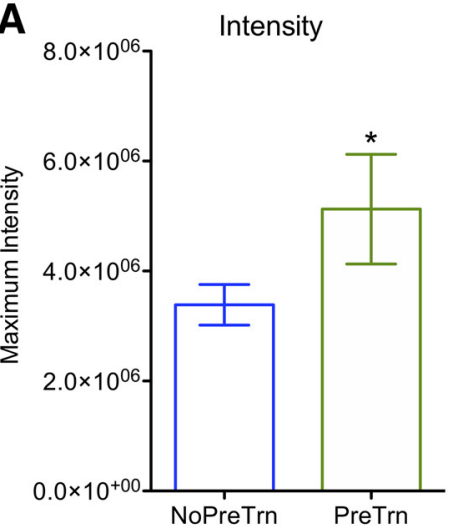

B

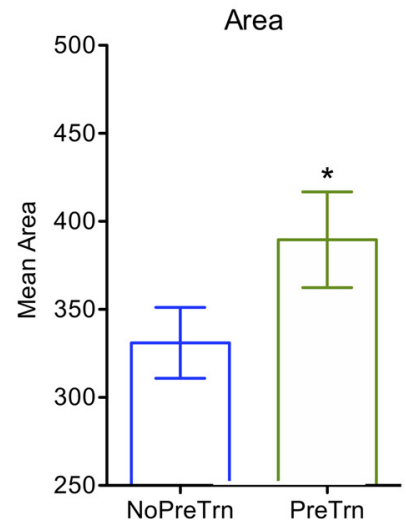

C
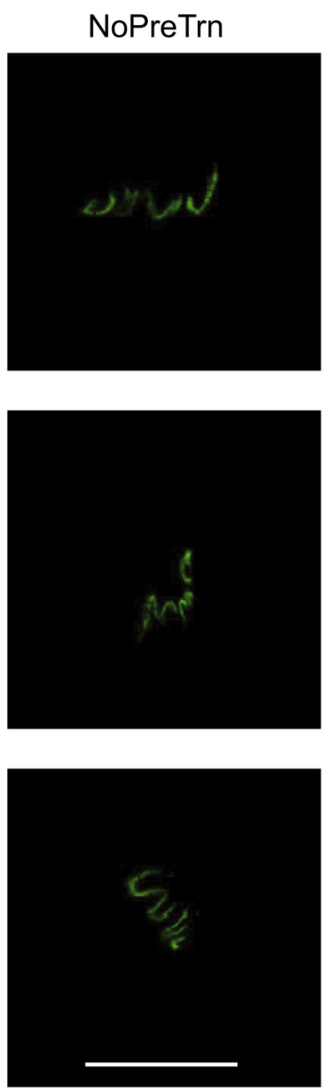

PreTrn
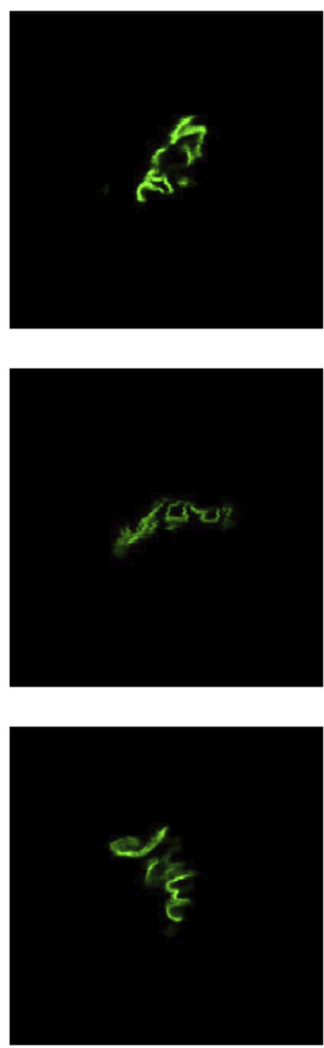

Figure 8. Instrumental training increased the intensity and area of AChR fluorescent labeling at the NMJ. The mean intensity $(\boldsymbol{A})$ and area $(\boldsymbol{B})$ of AChR labeling observed in the tibialis anterior muscles from the pretrained (PreTrn) or untrained (NoPreTrn) leg $(n=6)$. Error bars indicate SE. C, Representative images illustrating AChR labeling at the NMJ from the untrained (NoPreTrn) and trained (PreTrn) leg. Scale bar, $50 \mu \mathrm{m}$. Asterisks indicate $p<0.05$.

the end of training, the tibialis anterior muscles were removed from both the trained and untrained hind leg under pentobarbital anesthesia and prepared for sectioning. The AChR was labeled using Alexa-488- $\alpha$-bungarotoxin which, when illuminated with blue light, yields a green image. Quantitative fluorescence imaging was then used to assess the area and intensity of binding. For each muscle, all of the NMJs were identified and 10 were randomly selected for analysis. At each location, our analyses focused on labeling within a $200 \times 320$ pixel region that encompassed the NMJ. For each animal, the relative labeling observed in the untrained leg served as an internal (within-subjects) control. The design involved a single group $(n=6)$ and a within-subjects comparison between the trained (PreTrn) and untrained (NoPreTrn) leg. An additional 8 rats were used to evaluate the effect of spinal transection, per se, on AChR density. Four animals underwent a spinal transection while the other four received a sham surgery. The next day, one tibialis anterior muscle was collected from each animal and sectioned/labeled as described above.

As expected, exposure to response-contingent shock produced an increase in flexion duration [43.64 \pm 3.61 (mean \pm $\mathrm{SE})]$ in the trained leg $\left(F_{(5,29)}=1.98, p=0.0047\right)$, and elicited no response in the untrained (contralateral) leg $(0.0 \pm 0.0)$.

Labeling of the AChR was increased (intensity and area) in the tibialis anterior muscle from the trained leg (Fig. 8). Quantitative fluorescence imaging showed that training did not have a significant effect on the depth of labeling, as indexed by the number of scans required to encompass the receptor (all $F$ values $<1.0, p>0.05$ ). The average area of labeling (Fig. $8 A$ ) was higher in the trained leg $\left(F_{(1,11)}=7.08, p<0.0222\right)$. So too was the maximum intensity of labeling $\left(F_{(1,11)}=5.52, p=0.0386\right)$ (Fig. $\left.8 B\right)$. Training also produced a general increase in the intensity of labeling that approached significance $\left(F_{(1,11)}=4.21, p=0.0648\right)$.

To assess whether spinal transection, per se, induced an acute change in AChR labeling, we also compared muscle tissue from sham-operated and transected animals. No significant differences were observed (all $F$ values $<1.0, p>0.05$; data not shown).

The results demonstrate that behavioral training increases the area and maximum intensity of labeling for $\alpha$-bungarotoxin at the NMJ, implying an increase in AChR density. This peripheral modification would be expected to enhance the postsynaptic response to ACh release and thereby promote the maintenance of a flexion response.

\section{Peroneal stimulation substitutes for spinal input to generate learning}

Our results suggest that spinal neurons are essential to learning about the relation between a behavioral response (leg position) and an outcome (noxious stimulation). Prior work suggests that this learning is reinforced by the onset, rather than the offset, of electrical stimulation (Grau et al., 1998). This implies that learning depends on the relation between proprioceptive cues and 
shock onset (Fig. 1B). The consequence of this learning is a behavioral modification (an increase in flexion duration) that minimizes net shock exposure. We assume here that learning drives the motoneuron and $\mathrm{ACh}$ release at the NMJ, strengthening the elicited response and initiating processes that support the performance of the flexion response over time. Efferent neural output could, in combination with tibialis anterior muscle stimulation, induce an LTP-like process. Indeed, in vitro studies of Xenopus nervemuscle cultures have shown that nerve stimulation, in conjunction with a postsynaptic depolarization, can induce LTP at the NMJ (Wan and Poo, 1999). If this type of modification underlies the strengthening of the flexion response, we should be able to generate learning through a form of substitution by artificially driving the motoneuron output whenever electrical stimulation is applied to the tibialis anterior muscle. We evaluated this possibility by cutting communication with the spinal cord through a sciatic nerve transection and then stimulating the peroneal nerve, the branch of the sciatic nerve that innervates the tibialis anterior muscle and elicits a flexion response. The novel prediction was that stimulating both peroneal nerve and muscle would drive a learning-like increase in flexion.

A day after rats received a spinal cord transection, the right sciatic nerve was exposed and transected. An hour later, the peroneal nerve of the same leg was fitted with stimulating electrodes. Animals were then placed in the restraining tube, had electrodes implanted into the tibialis anterior muscle, and the contact electrode depth adjusted, as described above. One-third of the rats (Nerve+Muscle) then received 30 min of training with stimulation to both the peroneal nerve and tibialis anterior muscle whenever the contact electrode touched the underlying salt solution. Another third received response-contingent stimulation to just the tibialis anterior muscle (Muscle). The last third received response-contingent stimulation to only the peroneal nerve (Nerve). The experimental design involved three groups (Nerve, Muscle, or Nerve+Muscle) and required 18 animals $(n=6)$. The training data were analyzed across time (min 1-30), which served as a within-subjects variable.

As reported above, cutting the sciatic nerve blocked learning when rats received response-contingent shock to the tibialis anterior (Muscle) alone (Fig. 9A). Similarly, peroneal stimulation alone (Nerve) did not induce an increase in flexion duration. However, when both forms of stimulation were combined (Nerve + Muscle), rats exhibited a progressive increase in flexion duration. An ANOVA confirmed that there was a significant effect of training condition $\left(F_{(2,15)}=15.73, p=0.0002\right)$. There was also a significant effect of time bin and a time $\times$ training condition interaction (both $F$ values $>3.19, p<0.0086$ ). Post hoc comparisons of the group means (Fig. $9 B$ ) showed that the animals trained with Nerve+Muscle stimulation differed from the groups given stimulation to the muscle or nerve alone $(p<0.05)$. No other group difference approached statistical significance $(p>0.05)$.

Our results show that paired stimulation of the peroneal nerve and the tibialis anterior muscle is sufficient to drive an increase in flexion duration, our index of learning. Most importantly, this learning occurred although communication with the spinal cord had been removed.

\section{Paired stimulation induces an increase in flexion duration and EMG}

Having removed the spinal cord from the equation by cutting the sciatic nerve, we have focused on local events: the pairing of peroneal nerve activity with tibialis anterior stimulation. At the level of the NMJ, these events are posited to drive the peripheral alteration(s) that underlie the strengthening of the behavioral response. From this perspective, it should not be necessary to present the stimulation in a response-contingent manner, dependent on leg position. We should be able to drive a behavioral change by simply pairing peroneal stimulation with electrical stimulation of the tibialis anterior muscle using parameters that emulate the temporal distribution of stimulation within an instrumental paradigm. The present experiment evaluates this possibility by pairing peroneal stimulation with tibialis anterior stimulation using a Pavlovian paradigm, with 180 stimuli given in a paired or unpaired manner at a frequency of 0.1 or $0.5 \mathrm{~Hz}$. The effect of training was evaluated by measuring its impact on leg position and the EMG response elicited by peroneal stimulation.

A day after rats received a spinal cord transection $(N=24)$, the sciatic nerve was cut and the peroneal nerve was fitted with stimulating electrodes. Electrodes were then inserted into the tibialis anterior muscle, and the animal was placed into the restraining tube and marked for kinematic tracking. Next, the baseline EMG response to peroneal stimulation was recorded. Rats then received three AC shocks $(0.6 \mathrm{~mA}, 80 \mathrm{~ms})$ to the tibialis anterior muscle, and the resting position was recorded. Half the animals then received nerve and muscle stimulation in a paired manner for 180 trials, at 0.1 or $0.5 \mathrm{~Hz}$. The remaining rats received the stimuli in an unpaired manner. The full design involved a 2 (Paired vs Unpaired $) \times 2(0.1 \mathrm{vs} 0.5 \mathrm{~Hz})$ factorial $(n=6)$. In all conditions, leg position was recorded using a video camera and assessed after every 30 shocks to the muscle. At the end of training, the EMG response elicited by peroneal stimulation was tested. To evaluate whether training had a lasting effect, EMG was reassessed $30 \mathrm{~min}$ later. The average evoked response before training (Baseline) was computed by averaging the EMG signal 

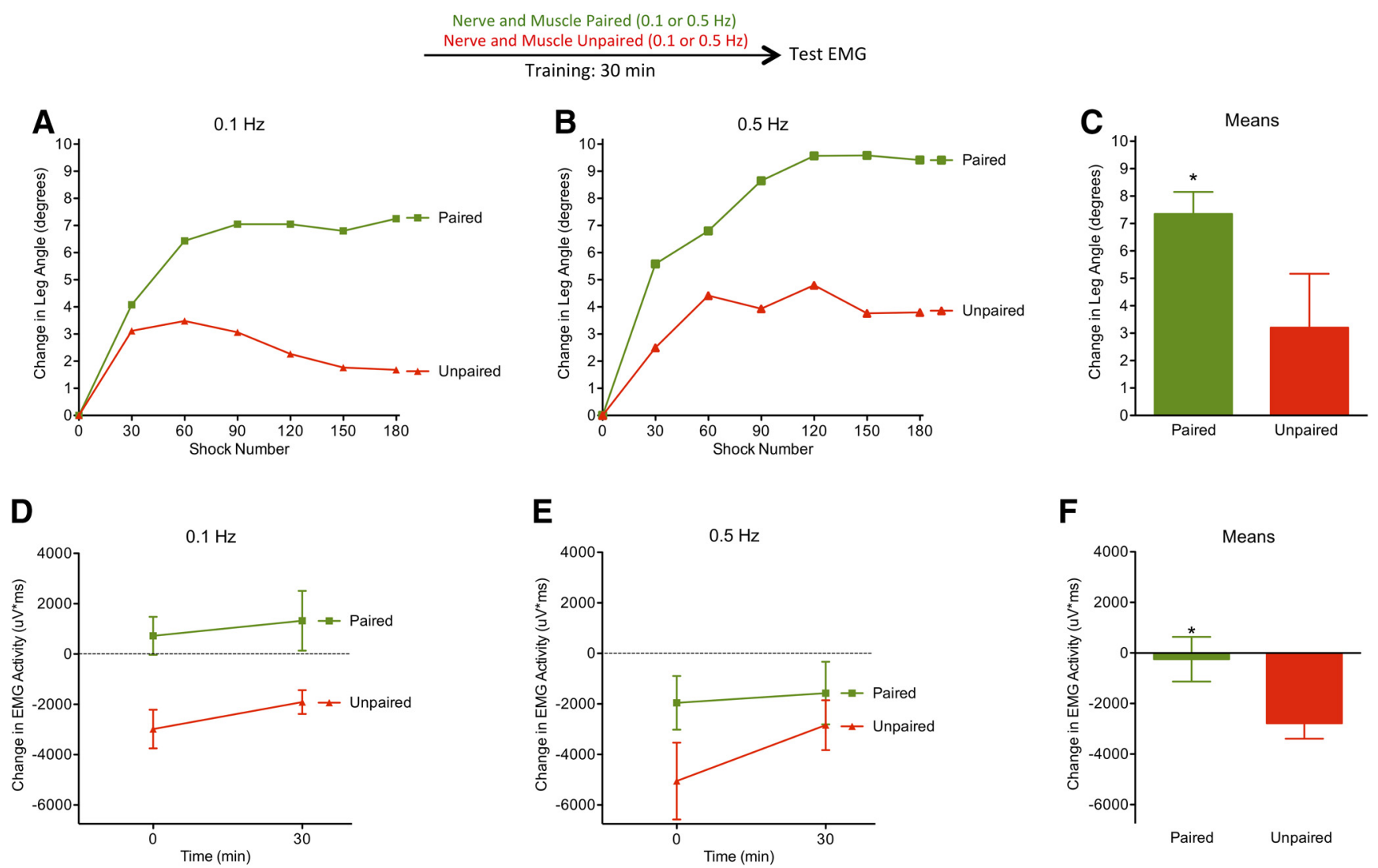

Figure 10. In animals that have received a sciatic cut, stimulating the peroneal nerve and the tibialis anterior muscle in a paired, but not an unpaired, manner induces a learning-like change in leg angle and increases the evoked EMG response. Paired stimulation of the tibialis anterior muscle and the peroneal nerve at $0.1(\boldsymbol{A})$ or $0.5(\boldsymbol{B}) \mathrm{Hz}$ induced a change in leg angle, relative to animals that received the same stimuli in an unpaired manner $(n=6)$. C, Mean performance during the 30 min test session. The EMG response elicited by stimulating the peroneal nerve was tested immediately after training $(0 \mathrm{~min})$ or $30 \mathrm{~min}$ after training with the stimuli presented at $0.1(\boldsymbol{D})$ or $0.5(\boldsymbol{E}) \mathrm{Hz}$. Peroneal stimulation elicited greater EMG activity in animals that had received the stimuli in a paired manner. Increasing the frequency of stimulation caused a general reduction (habituation) in EMG activity. $\boldsymbol{F}$, The mean change in EMG activity, collapsed across training frequency and test time. Error bars indicate SE. Asterisks indicate $p<0.05$.

across the three test pulses and time $(100 \mathrm{~ms})$. The mean response at 0 and $30 \mathrm{~min}$ after training was computed in the same way, and time ( 0 vs $30 \mathrm{~min}$ ) served as a within-subjects variable. To control for variation in EMG amplitude across subjects, an ANCOVA was used to analyze the post-training data, with pretraining EMG activity serving as the covariate.

Before training, there were no group differences in the EMG response evoked by stimulation of the peroneal nerve (all $F$ values $<1.0, p>0.05$ ).

Paired, but not unpaired, stimulation of the peroneal nerve and tibialis anterior muscle induced a change in leg angle that emulated the effect of training with controllable stimulation, and this was true independent of whether the stimuli were presented at 0.1 (Fig. $10 A$ ) or $0.5 \mathrm{~Hz}$ (Fig. 10B). An ANOVA confirmed that stimulation changed leg angle over time and that the magnitude of this effect depended on whether the stimuli were presented in a paired or unpaired manner (both $F$ values $>3.14$, $p<0.0112)$. Neither the main effect of frequency, nor its interaction with other factors, was statistically significant (all $F$ values $<$ $2.87, p>0.05)$.

Repeated stimulation led to a slight reduction (habituation) in the EMG elicited by peroneal stimulation in animals that received the stimuli at a higher frequency $(0.5 \mathrm{~Hz})$ (Fig. 10D,E). More importantly, a stronger response was observed in rats given the stimuli in a paired manner, and this was true independent of training frequency. Further, an effect was still evident when animals were retested 30 min later. An ANCOVA confirmed that a weaker response was observed across groups after $0.5 \mathrm{~Hz}$ stimulation $\left(F_{(1,19)}=4.49, p=0.0476\right)$. More importantly, pairing the stimuli had a significant impact $\left(F_{(1,19)}=7.62, p=0.0124\right)$, and the magnitude of this effect did not vary across stimulus frequency $\left(F_{(1,19)}<1.0, p>0.05\right)$. Further, similar results were obtained at both the immediate and delayed $(30 \mathrm{~min})$ test points (all $F$ values $<1.80, p>0.05$ ).

We found that paired stimulation of the peroneal nerve and tibialis anterior muscle produced an increase in the flexion response relative to animals that received the same stimuli in an unpaired manner. Likewise, experience with paired stimulation increased the EMG response elicited by peroneal stimulation, relative to animals that received the same stimuli in an unpaired manner. These findings show that local events can drive a physiological alteration at the NMJ and that this experience has a lasting effect.

Evidence that glutamatergic signaling plays an essential role Our work suggests that learning drives a form of Hebbian LTP that increases AChR density at the NMJ, yielding functional changes akin to those implicated in NMDAR-mediated LTP (Malinow and Malenka, 2002). At the NMJ, the development of LTP has been tied to receptor-mediated $\mathrm{Ca}^{2+}$ influx (RMCI) (Ouanounou et al., 2016). Interestingly, this $\mathrm{Ca}^{2+}$ flow could be mediated by the activation of NMDARs, which are found on the 

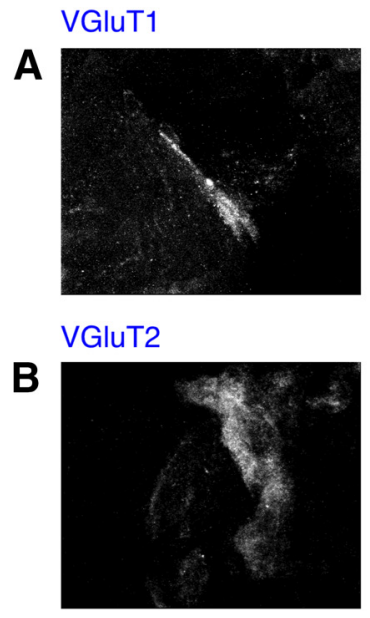

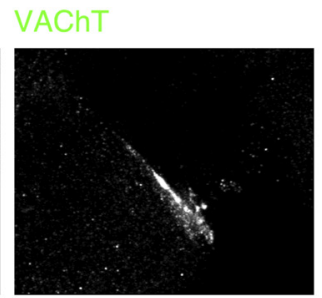

$\mathrm{AChR}$

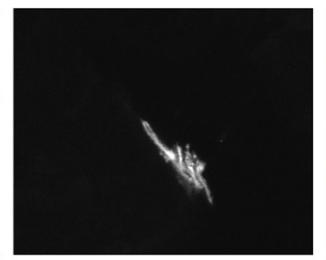

VAChT

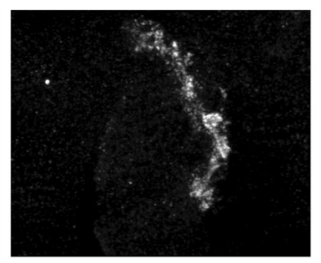

AChR

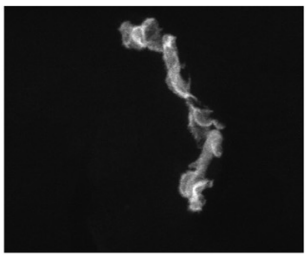

Combined

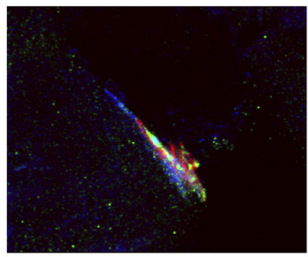

Combined

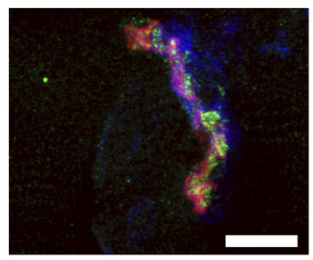

Figure 11. VGluT, VAChT, and AChR immunostaining at the NMJ of the tibialis anterior muscle. Images were obtained with a FV1000 confocal microscope equipped with $100 \times / 1.4$ oil immersion objective and were saved in a 16 bit grayscale format. Each row represents VGluT, VAChT, and AChR labeling at an individual NMJ. The combined images show that VGluT (blue) is colocalized with VAChT (green) within the presynaptic terminal, apposed to the AChR (red). $\boldsymbol{A}$, Colabeling with VGluT1. B, Colabeling with VGluT2. Scale bar, $10 \mu \mathrm{m}$.

postsynaptic membrane of vertebrate skeletal muscles closely associated with AChRs in the depths of the secondary folds (Berger et al., 1995; Mays et al., 2009; Malomouzh et al., 2011). Further, presynaptic terminals from hindlimb muscles are positive for glutamate, which may be coreleased with ACh (Waerhaug and Ottersen, 1993). Glutamate immunoreactivity appears to be higher in motoneuron neuron terminals that innervate fast twitch (Type II) muscles, where they demonstrate levels comparable to that observed within glutamatergic regions of the hippocampus (Waerhaug and Ottersen, 1993). The idea that motoneurons can corelease glutamate was reinforced by studies demonstrating labeling for VGluTs, both within the spinal cord (Herzog et al., 2004; Nishimaru et al., 2005) and the motor endplate (Boulland et al., 2004; Ewald et al., 2006; Bertuzzi et al., 2018). Because the tibialis anterior muscle contains a high proportion of fast fibers (Pullen, 1977), we hypothesized that motor neurons that innervate this muscle may also express VGluT. Supporting this, immunostaining revealed labeling for VGluT1 and VGluT2 in the lower motor neuron junctions colocalized with VAChT (Fig. 11).

It has been suggested that the corelease of glutamate from motoneurons modulates ACh function (Li and Harlow, 2014; Colombo and Francolini, 2019). Supporting this, engaging the NMDAR on muscle fibers has been shown to reduce the resting membrane potential (Petrov et al., 2013) and contribute to the selection of synaptic inputs during development (Personius et al., 2016). Glutamate release at the NMJ can be triggered by moderate- to high-frequency motoneuron stimulation (Slater, 2015; Kasimov et al., 2017) and appears to contribute to functional recovery after nerve injury (Brunelli et al., 2005; Pizzi et al., 2006; Francolini et al., 2009). Interestingly, injury and exercise can induce a phenotypic shift in a subclass (reserve pool) of motoneurons that innervate fast muscles, bringing about an enhancement in motor function that can be blocked by pretreatment with an NMDAR antagonist (Bertuzzi et al., 2018). These observations led Bertuzzi et al. (2018) to suggest that glutamatergic signaling provides a kind of embedded plasticity that enables adaptability to new environmental demands. We hypothesize that training with response-contingent shock drives an increase in flexion duration by amplifying the motoric output at shock onset. If this occurs, glutamatergic signaling at the NMJ of the tibialis anterior muscle could provide a form of coincidence detection, yielding Hebbian-based plasticity that drives and maintains the increase in flexion duration. We explored this possibility by testing whether intramuscular application of a NMDAR antagonist (MK801) disrupts the acquisition and maintenance of the learned response.

We first examined whether intramuscular MK801 (Tocris Bioscience) affected acquisition in spinally transected rats given electrical stimulation to the tibialis anterior muscle whenever the hind leg was extended. A day after transection, MK801 (50 or $200 \mu \mathrm{g}$, dissolved in $1 \mu \mathrm{l}$ of $0.9 \%$ saline) or its vehicle was microinjected into the tibialis anterior muscle. Twenty-five minutes later, animals were tested with response-contingent shock as described above. The design involved three groups (drug dose) with 6 animals per condition. The training data were analyzed across time ( $\min 1-30)$, which served as a within-subjects variable.

As expected, vehicle-treated rats given response-contingent shock learned to maintain the stimulated leg in a flexed position (Fig. 12A). Pretreatment with MK801 disrupted learning in a dose-dependent manner. An ANOVA revealed a significant effect of drug treatment and time (both $F$ values $>2.387, p<$ 0.0001). Post hoc comparisons of the group means (Fig. 12B) revealed that the group treated with $200 \mu \mathrm{g}$ of MK801 differed from the other two $(p<0.05)$ and that the $50 \mu$ g treated group did not differ from the vehicle controls $(p>0.05)$. MK801 did not disrupt the capacity to perform a flexion response (Fig. 12C; all $F$ values $<1.289, p>0.05$ ).

We then assessed the effect of intramuscular MK801 on the maintenance of the learned response using a design analogous to that used to assess the effect of lidocaine and curare. A day after spinal transection, animals were placed in the test apparatus and set up for stimulation of the tibialis anterior muscle. Half the animals then received 30 min of training with controllable shock (PreTrn). The remaining animals served as the no pretraining (NoPreTrn) controls. After $25 \mathrm{~min}$, half of the rats in each condition received an intramuscular injection of MK801 $(200 \mu \mathrm{g})$. The remaining rats were treated with the vehicle. Five minutes later, all of the animals underwent $30 \mathrm{~min}$ of testing with responsecontingent shock. The full design involved a 2 (PreTrn or NoPreTrn) $\times 2$ (MK801 or Veh) factorial $(n=14$; two 

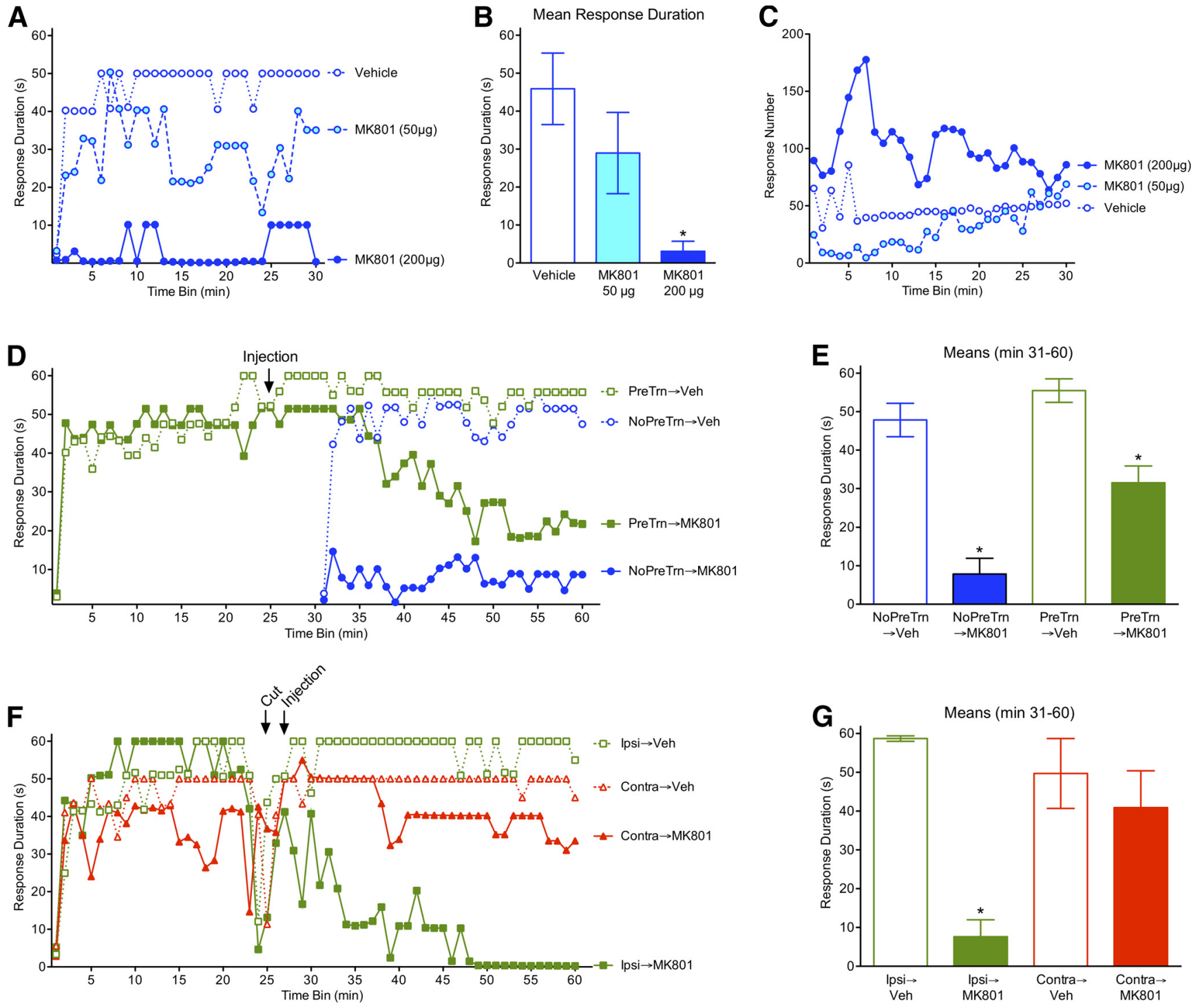

Figure 12. Intramuscular application of the NMDAR antagonist MK801 interfered with the acquisition and maintenance of the instrumental response. $\boldsymbol{A}$, Spinally transected rats received an intramuscular injection of MK801 (50 or $200 \mu \mathrm{g}$ ) or its vehicle before being trained for $30 \mathrm{~min}$ with response-contingent shock $(n=6)$. Vehicle-treated rats exhibited a progressive increase in response duration indicative of learning. Pretreatment with MK801 disrupted learning. $\boldsymbol{B}$, Mean response duration during the 30 min of training. $\boldsymbol{C}$, Pretreatment with MK801 did not disrupt the capacity to perform a flexion response. $\boldsymbol{D}$, Rats received $30 \mathrm{~min}$ of training with response-contingent shock (PreTrn) or nothing (NoPreTrn) and an intramuscular injection at $25 \mathrm{~min}(n=14)$. Rats that were not pretrained and given vehicle (NoPreTrn $\rightarrow$ Veh) exhibited an increase in response duration when tested with response-contingent shock. Minus pretraining, MK801 blocked learning (NoPreTrn $\rightarrow$ MK801). Treatment with MK801 also undermined the maintenance of the learned response in pretrained animals (PreTrn $\rightarrow$ MK801). $E$, Mean performance during testing (min 31-60). Error bars indicate SE. $\boldsymbol{F}$, All groups were trained for 30 min with response-contingent shock. They then received a sciatic cut, followed by an intramuscular injection of MK801 or its vehicle (Veh) applied on the same leg (Ipsi) or the opposite (Contra) leg $(n=8)$. MK801 disrupted the maintenance of the learned response when applied to the pretrained leg (Ipsi $\rightarrow$ MK801), but had no effect when given to the opposite (untrained) leg (Contra-MK801). Error bars indicate SE. G. Mean performance during testing (min 31-60). Error bars indicate SE. Asterisks indicate $p<0.05$.

replications). Data from the training (min 1-30) and test phases (min 31-60) were analyzed separately, with time bin serving as a within-subjects variable.

Rats that were pretrained with response-contingent shock exhibited an increase in flexion duration (Fig. $12 D ; F_{(29,754)}=$ 10.198, $p<0.0001)$. During the subsequent 30 min test session, animals that were not pretrained before MK801 treatment (NoPreTrn $\rightarrow$ MK801) failed to learn. MK801 also disrupted the maintenance of the instrumental response in pretrained rats (PreTrn $\rightarrow$ MK801). An ANOVA revealed significant main effects of drug treatment, training condition, and time (all $F$ values $>$ 2.403, $p<0.004)$. In addition, the interactions between time and training condition, drug treatment, and the combination of training and drug treatment were all statistically significant (all $F$ values $>1.960, p<0.0018$ ). The three-way interaction indicates that the change in response duration observed over time depends on both training and drug treatment. The drug $\times$ training interaction was not significant $\left(F_{(1,52)}=3.719, p>0.05\right)$. Post hoc comparisons of the group means (Fig. 12E) showed that the two MK801-treated groups differed from each other and both of the vehicle-treated groups $(p<0.05)$. No other comparisons were significant $(p>0.05)$.

It could be argued that MK801 diffused out of the muscle tissue and had a systemic effect, potentially impacting central neurons that contribute to the maintenance of the learned response. Alternatively, local application of the drug could engage sensory 
fibers, generating an aberrant afferent signal that drives the loss of the learned response. We addressed both of these alternatives by comparing the effect of MK801 applied to either the ipsilateral (Ipsi) or contralateral (Contra) tibialis anterior muscle in animals that received a sciatic transection at the end of training. Administration of MK801 to the contralateral leg provides a control for drug diffusion. Cutting the sciatic nerve on the trained leg before drug administration removes the possibility of an indirect, spinally dependent effect on the learned response.

Spinally transected rats underwent 30 min of training with response-contingent shock. After $25 \mathrm{~min}$, the sciatic nerve on the trained leg was cut as described above. Immediately after, animals received either MK801 $(200 \mu \mathrm{g})$ or the vehicle (Veh). Animals were then tested for $30 \mathrm{~min}$ with response-contingent shock. The full design involved a 2 (MK801 or Veh) $\times 2$ (Ipsi or Contra) factorial with 8 animals per group. Data from the training (min 1-30) and test phases (min 31-60) were analyzed separately, with time bin serving as a within-subjects variable.

During pretraining (Fig. 12F), rats exhibited a progressive increase in flexion duration $\left(F_{(29,580)}=7.614, p<0.0001\right)$. There were no group differences before testing (all $F$ values $<1.0$, $p>0.05)$. Animals that received the vehicle maintained the learned response across the $30 \mathrm{~min}$ of testing. Application of MK801 to the pretrained leg (MK801 $\rightarrow$ Ipsi) disrupted performance of the instrumental response. Application of MK801 to the opposite (MK801 $\rightarrow$ Contra) leg had no effect. An ANOVA confirmed that there was a significant effect of drug treatment $\left(F_{(1,20)}=15.552, p<0.0008\right)$ and that the effect of MK801 depended on whether it was administered on the ipsilateral or contralateral side $\left(F_{(1,20)}=7.765, p=0.0114\right)$. Treatment with MK801 on the ipsilateral side caused a decline in performance over the $30 \mathrm{~min}$ of testing, yielding a significant effect of time and a time $\times$ drug treatment interaction (both $F$ values $>1.994$, $p<0.0017)$. A comparison of the group means from the $30 \mathrm{~min}$ test period (Fig. 12G) showed that the group given MK801 on the ipsilateral side $(\mathrm{MK} 801 \rightarrow \mathrm{Ipsi})$ differed from the other three $(p<0.05)$. No other group comparison was significant $(p>$ $0.05)$.

It has been suggested that engaging postsynaptic NMDARs could influence ACh release by means of nitric oxide (NO), which can function as a retrograde transmitter (Grozdanovic and Gossrau, 1998; Ribera et al., 1998; Pinard and Robitaille, 2008). Supporting this, the enzyme needed to synthesize NO (nitric oxide synthase) is colocalized with NMDARs in skeletal muscles. Given these observations, we tested whether intramuscular administration of the NO-synthase inhibitor L-NAME $(0,3$, or $10 \mathrm{mg})$ affects the acquisition of the learned response. L-NAME had no effect (both $F$ values $<1.0, p>0.05$; data not shown).

\section{Intramuscular MK801 disrupts the learning-induced increase in EMG activity}

We found that peripheral application of MK801 to the tibialis anterior muscle disrupts the acquisition and maintenance of the learned response. Importantly, we reinforced these observations by showing that MK801 has the same outcome after the sciatic nerve is cut and that application of the drug to the opposite leg has no effect. To reinforce these observations, we assessed the impact of intramuscular MK801 on the electrical signal elicited by stimulation of the sciatic nerve using EMG in spinally transected rats that had received controllable (Master) or uncontrollable (Yoked) stimulation.
A day after rats received a spinal transection, the sciatic nerve was exposed, a nerve cuff was positioned on the sciatic nerve, and EMG electrodes were inserted into the tibialis anterior muscle, as described above. Next, pretraining (baseline) electrical activity was assessed, and animals were set up for instrumental training. Half of the rats (Master) then received 30 min of training with response-contingent shock. The remaining animals received the same distribution of shock but independent of leg position (Yoked). At the end of training, the sciatic nerve was transected, and animals received an intramuscular injection of MK801 $(200 \mu \mathrm{g}$ in $300 \mu \mathrm{l})$ or vehicle (Veh). Finally, the EMG response elicited by sciatic stimulation was reassessed. The full design involved a 2 (Master or Yoked) $\times 2$ (MK801 or Veh) factorial $(n=6)$.

Before training, the EMG response elicited by stimulation of the sciatic nerve $(1 \mathrm{~V})$ did not differ across groups (all $F$ values $<1.0, p>0.05$ ). As expected, exposure to controllable shock (Master), but not uncontrollable shock (Yoked), elicited an increase in flexion duration across the 30 min of training (Fig. 13A). An ANOVA confirmed that the main effects of training condition, time, and their interaction were all statistically significant (all $F$ values $>4.413, p<0.0001$ ). No other term approached statistical significance (all $F$ values $<1.446, p>0.05$ ).

At the end of training, stimulation of the sciatic nerve elicited a stronger electrical signal within the tibialis anterior muscle in Master rats treated with the vehicle (Master $\rightarrow \mathrm{Veh}$ ), relative to animals in the Yoked condition (Yoked $\rightarrow$ Veh) (Fig. 13B-E). This effect of training was eliminated by MK801. To control for variation in baseline EMG activity, an ANCOVA was performed using the pretraining response as a covariate. This analysis revealed a main effect of training condition $\left(F_{(1,19)}=22.641\right.$, $p<0.0001)$ and drug treatment $\left(F_{(1,19)}=10.132, p=0.0049\right)$. Importantly, the effect of training depended on drug treatment $\left(F_{(1,19)}=11.657, p=0.0029\right)$. The main effect of time and the interactions with training condition and drug treatment were also significant (all $F$ values $>2.297, p<0.0185$ ). Post hoc comparisons of the group means showed that the Master rats treated with the vehicle differed from the other three $(p<0.05)$. No other group comparison was significant $(p>0.05)$.

The findings reinforce our earlier observation that training with controllable stimulation bolsters the evoked electrical response within the muscle. The training-induced augmentation of the evoked response was eliminated by administration of the NMDAR antagonist MK801, and this was observed in rats that had undergone a sciatic transection, again mitigating an indirect (spinally dependent) effect of drug treatment. Importantly, these effects were observed using the lowest effective dose of MK801 (Fig. 12A). Further, drug treatment did not eliminate the evoked EMG or muscle contraction, which argues against a nonspecific effect. Future experiments may expand on these observations using antagonists that target alternative forms of the NMDAR.

\section{Discussion}

\section{Evidence the CNS can induce a modification at the NMJ}

Prior research motivated a model of spinal learning (Fig. 1B) that assumed the maintenance of the behavioral response was tied to a neural modification within the spinal cord that drives the efferent motor output. Yet, after animals learned to maintain a hindlimb in a flexed position, inactivating the lumbosacral spinal cord, excising this tissue, or blocking peripheral communication by cutting the sciatic nerve had no effect on the maintenance of the behavioral response. These observations imply that 



Figure 13. Instrumental training augmented the EMG response elicited by sciatic stimulation, and this effect was blocked by MK801. Spinally transected rats received 30 min of training with response-contingent electrical stimulation to the tibialis anterior muscle (Master) or an equivalent amount of stimulation independent of leg position (Yoked). At the end of training, the sciatic nerve was transected, and rats received an intramuscular injection of MK801 or its vehicle (Veh). The electrical response elicited by sciatic stimulation was then assessed using EMG $(n=6)$. A, Before drug infusion, animals that received $30 \mathrm{~min}$ of training with response-contingent (Master) leg shock exhibited an increase in flexion duration relative to rats that received an equivalent exposure to shock independent of leg position (Yoked). $\boldsymbol{B}$, Instrumental training augmented the EMG response elicited by stimulation of the sciatic nerve in vehicle-treated Master rats, relative to the Yoked controls, and this effect was blocked by MK801. C, The mean change in EMG activity collapsed across the testing period. Examples of the EMG response elicited in Master animals that received the vehicle $(\boldsymbol{D}$, Master $\rightarrow$ Veh $)$ or MK801 $(\boldsymbol{E}$, Master $\rightarrow$ MK801). Error bars indicate SE. Asterisks indicate $p<0.05$.

training induces a peripheral modification that contributes to the maintenance of the learned response. This conclusion was reinforced with evidence that exposure to controllable, but not uncontrollable, stimulation augments evoked EMG activity and that this effect survives a sciatic cut.

The training-induced increase in flexion duration was eliminated by intramuscular curare, which implicates ACh activity. When curare was given after flexion force was adjusted to the usual level, performance was disrupted, which suggests that electrical stimulation of the muscle elicits presynaptic neural activity and $\mathrm{ACh}$ release. While this stimulation appeared to have some capacity to directly activate muscle fibers, this component was relatively weak and quickly declined (habituated) with repeated stimulation. Additional evidence that ACh activity plays a critical role was obtained using fluorescent imaging, which showed that training increases the area and intensity of $\alpha$-bungarotoxin binding, implying a trafficking of AChRs to the NMJ.

Based on these observations, we hypothesized that efferent motor output from the spinal cord, in conjunction with stimulation of the tibialis anterior muscle, induces a modification at the 
A

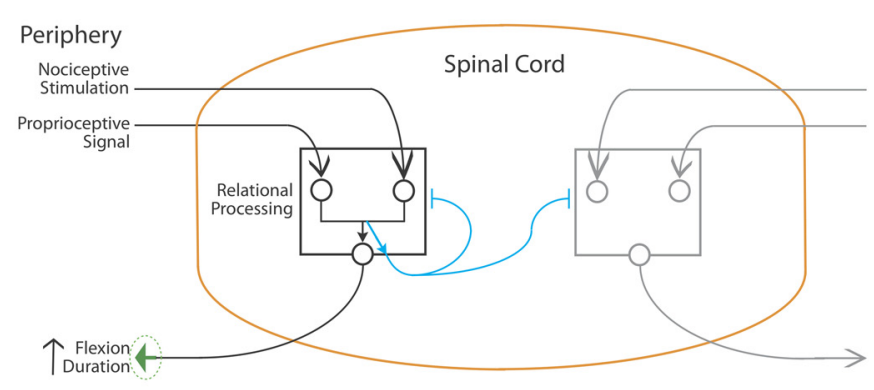

B

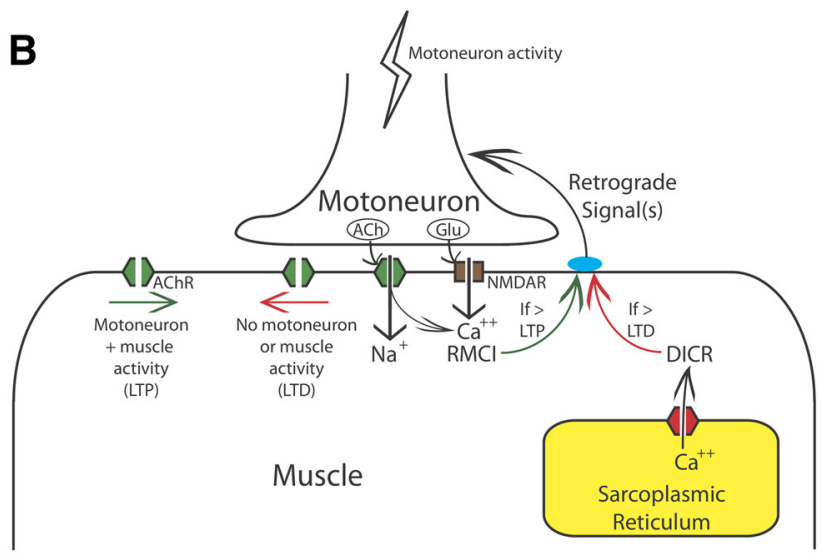

\section{ACh release}

\begin{tabular}{ccc}
$\begin{array}{c}\text { Motoneuron } \\
\text { activity \& RMCl }\end{array}$ & $\begin{array}{c}\text { Muscle } \\
\text { activity (DICR) }\end{array}$ & $\begin{array}{c}\text { Change in } \\
\text { transmitter release }\end{array}$ \\
\hline Moderate & Weak & Increase (LTP) \\
Moderate & Moderate & No change \\
\hline Weak & Strong & Decrease (LTD) \\
\hline In development & &
\end{tabular}

\section{AChR trafficking}

\begin{tabular}{ccc}
$\begin{array}{c}\text { ACh triggered } \\
\mathrm{Ca}^{++} \text {entry }(\mathrm{RMCl})\end{array}$ & $\begin{array}{c}\text { Muscle } \\
\text { activity (DICR) }\end{array}$ & $\begin{array}{c}\text { AChR in } \\
\text { active region }\end{array}$ \\
\hline Yes & Yes & Increase (LTP) \\
No & Yes & No change \\
\hline No & No & Decrease (LTD) \\
\hline After nerve injury & &
\end{tabular}

Figure 14. Relational processing within the spinal cord guides a peripheral modification at the NMJ that maintains the behavioral response. $A$, It is assumed that spinal neurons are sensitive to the relation between a nociceptive stimulus and proprioceptive signals related to limb position. As a consequence of this relation, an efferent motoneuron output is generated that leads to LTP, and an upregulation of the AChR, at the NMJ. The modification at the NMJ (green triangle) maintains the target response. In addition, relational processing engages a mechanism (blue) that affects how subsequent stimuli are processed. Experience with controllable stimulation enables processing, and this effect extends to stimuli applied to the contralateral leg (gray lines and box). $B$, A schematic representation of the processes that guide alterations in presynaptic release and the trafficking of the AChR. ACh release from the presynaptic neuron would activate AChRs (green hexagons), which enables cation entry and the depolarization of the postsynaptic cell. It is assumed that training or exercise can induce a phenotypic shift in motoneuron function that enables the corelease of glutamate (Bertuzzi et al., 2018). Removing the $\mathrm{Mg}^{++}$block from the NMDAR (brown rectangle) in the presence of extracellular glutamate would enable $\mathrm{Ca}^{2+}$ entry, bolstering RMCI. Depolarization of the cell would also trigger $\mathrm{Ca}^{2+}$ release from the sarcoplasmic reticulum (DICR). The relative balance between presynaptic release/RMCl and DICR is thought to regulate how a retrograde signal(s) affects presynaptic release (Ouanounou et al., 2016). The trafficking of AChRs to/from the active zone is modulated the activation of the AChR (RMCI) and muscle activity (DICR). C, Muscle contractions (DICR) when motoneuron input is weak initiates processes that reduce transmitter release, inducing a form of LTD. Such a process may contribute to the loss of redundant motoneuron inputs during development. When motoneuron input/transmitter release is moderate and a weak muscular response (DICR) is elicited, transmitter release is enhanced (LTP). D, In the absence of ACh release and muscle activity (e.g., after nerve injury), AChRs are trafficked away from the active zone of the synapse (Akaaboune et al., 1999). Engaging the muscle with electrical stimulation (DICR) blocks this effect. Engaging ACh release (RMCI) and muscle activity (DICR) through training or exercise can increase AChR density at the NMJ.

NMJ that fosters the performance of a flexion response. To explore this possibility, we cut the sciatic nerve and applied stimulation to the branch (peroneal) that contains the motoneuron axons that innervate the tibialis anterior. When the nerve and muscle were stimulated together in a response-contingent or Pavlovian (paired) manner, animals exhibited an increase in flexion duration over time. Pairing likely matters because engaging the peroneal nerve would have a nonselective effect that drives efferent output to multiple muscles. Stimulating the tibialis anterior would selectively augment presynaptic activity in the relevant motoneuron, amplifying $\mathrm{ACh}$ release and driving the processes that lead to a stronger flexion response. From this perspective, coupling peroneal nerve and tibialis anterior stimulation induces a kind of pairing-specific enhanced sensitization wherein the nerve stimulation has a facilitatory effect (Walters and Byrne, 1983).

Further analysis provided evidence that glutamatergic signaling contributes to learning-induced plasticity at the NMJ. We showed that, in spinally transected rats, the transporters for glutamate (VGluT) and ACh (VAChT) are coexpressed within the presynaptic terminals of axons projecting to the NMJ, apposed to the postsynaptic AChR. Infusing the NMDA antagonist MK801 into the muscle before training blocked learning. Likewise, intramuscular MK801 disrupted the maintenance of the instrumental response and the training-induced augmentation of the evoked electrical response. Importantly, the latter outcomes were evident after communication with the spinal cord was cut by means of a sciatic transection, discounting the possibility that the effect of MK801 was mediated by a central mechanism. Our results suggest that learning induces a phenotypic shift in the efferent motoneuron output that drives glutamate release at the NMJ, engaging the NMDAR during the period of depolarization. Glutamate activation of the NMDAR, coincident with a strong depolarization, would drive a form of Hebbian plasticity, mediated by an enhancement in $\mathrm{Ca}^{2+}$ influx that activates processes (receptor trafficking, transmitter release) that augment the evoked response.

\section{Revising our model of learning}

Our results suggest that relational processing within the spinal cord guides a peripheral process that brings about a lasting change (a kind of memory) in behavior by augmenting the muscular response elicited by the efferent motoneuron. The general framework (Fig. 14A) is consistent with past theoretical work suggesting that spinal neurons are biologically tuned to detect the relation between a noxious input and leg position (Grau et al., 2012; Grau, 2014) and that the efferent output is gated by step cycle phase (Forssberg et al., 1977; Forssberg, 1979). From 
this perspective, the selection of the appropriate defensive response occurs in a mechanical manner executed through a biologically prepared circuit. If the system is prewired, noxious stimulus at a particular leg position should drive the appropriate efferent motoneuron output at the start of training. Supporting this, we found that instituting a behavioral relation impacts response duration and EMG activity within the first minute of training.

The findings encourage a new perspective regarding learning within the spinal cord, shifting the focus to the regulation of physiological circuits. We have shown that uncontrollable/ unpredictable stimulation can disable the capacity to learn, an effect we have related to an impairment in relational processing (Grau et al., 1998, 2006, 2020; Crown et al., 2002b; Ferguson et al., 2012a,b; Grau, 2014). Conversely, prior exposure to controllable/predictable stimulation can counter this effect and foster relational learning (Crown and Grau, 2001; Grau et al., 2012; Huie et al., 2012b). Both the induction and the expression of these consequences of learning are blocked by intrathecal drug manipulations (Ferguson et al., 2006, 2008; Vichaya et al., 2009; Huie et al., 2012a,b). Further, the outcomes are not limb-specific (Crown et al., 2002a; Joynes et al., 2003), and we showed here that the enabling effect of training depends on communication with the spinal cord. The implication is that new learning and the metaplastic enhancement of learning depend on intraspinal processes. The new framework (Fig. 14A) suggests that behavioral control may enable learning because it fosters relational processing and/or amplifies the efferent motoneuron output, to support a stronger flexion response when the response criterion is raised. Conversely, uncontrollable stimulation could undermine the capacity to learn by disrupting relational processing and/or the selective amplification of an adaptive motoneuron output.

The discovery that environmental relations can regulate the capacity to learn led us previously to posit an independent (metaplastic) process (Fig. 1B). The view suggested here ascribes the selection of the target response to relational processing. From this perspective, processes inherent to this mechanism could regulate long-term potential (Fig. 14A), to foster or inhibit neural circuits on the basis of environmental relations.

\section{Plasticity at the NMJ}

The idea that spinal processes guide a peripheral modification by driving presynaptic neural activity at the NMJ is consistent with studies examining plastic potential at the NMJ in neuron-muscle cultures and isolated muscles (Dan and Poo, 1992; Wan and Poo, 1999). This work has shown that repeated application of ACh can induce LTD through cellular processes that lead to a muscle contraction and, in particular, the release of $\mathrm{Ca}^{2+}$ from the sarcoplasmic reticulum (Dan and Poo, 1992). This DICR is hypothesized to trigger a retrograde signal that, in turn, leads to a reduction in quantal release from the presynaptic neuron (Fig. $14 B$ ). Interestingly, the development of LTD is modulated by presynaptic neural activity; if the motor axon is stimulated within a few milliseconds of ACh application, no LTD is observed (Dan and Poo, 1992).

Other work indicates that motor axon stimulation can induce a form of LTP at the NMJ (Fig. 14C), attributable to a rise in quantal release (Wan and Poo, 1999; Ouanounou et al., 2016). LTP can develop rapidly at the NMJ, and the degree of potentiation is inversely correlated with initial synaptic strength. Similar to LTD, the emergence of LTP depends on postsynaptic activity because the effect is blocked by pretreatment with curare. The development of LTP also requires presynaptic neural activity; engaging the AChR without presynaptic activity yields LTD rather than LTP (Dan and Poo, 1992). Our results suggest that the training-induced increase in flexion duration is related to ACh release, which gains the capacity to drive a stronger EMG response in the muscle. It is assumed here that the development of this acquired alteration depends on efferent drive from the spinal cord, which fosters the development of an LTP-like process when paired with muscle stimulation.

In addition, it appears that training can bring about an alteration in AChR density at the NMJ (Fig. 14D). This is consistent with past work demonstrating that muscle inactivity leads to a downregulation of the AChR (LTD) attributed to the migration of the AChR to the perisynaptic zone and eventual internalization (Akaaboune et al., 1999). A similar effect was observed after denervation. Interestingly, stimulation of the muscle (under conditions that avoided nerve activation) blocked this effect, which implies that it is linked to a lack of muscle activity (DICR). While stimulation prevented loss, it did not augment AChR density at the NMJ, implying that the enhancement reported above depends on both RMCI and a prolonged muscle contraction (linked to DICR; Fig. 14D). The result would be an amplification of the RMCI elicited by ACh release (LTP), an effect that would help to maintain both the flexion response and the requisite safety factor (Wood and Slater, 2001).

Our work suggests that paired activity of the motoneuron and muscle drives a form of Hebbian LTP that increases AChR density at the NMJ, linking this effect to a rise in RMCI. While prior work assumed that the requisite $\mathrm{Ca}^{2+}$ influx was linked to engaging AChRs (Ouanounou et al., 2016), our data suggest an alternative interpretation that ties plasticity at the NMJ to glutamatergic mechanisms that have been implicated in centrally mediated LTP (Tabone and Ramaswami, 2012; Colombo and Francolini, 2019). From this perspective, training and exercise can bring about a phenotypic shift that increases glutamate release and the activation of AMPARs and NMDARs on the postsynaptic cell at the NMJ (Bertuzzi et al., 2018; Mays et al., 2009). Engaging NMDARs would promote $\mathrm{Ca}^{2+}$ entry (RMCI), which could drive the development of LTP by enhancing transmitter release and receptor trafficking.

In conclusion, our results show that learning can induce a modification at the NMJ that helps to preserve a behavioral modification over time and that glutamatergic signaling contributes to this plasticity. The findings are consistent with in vitro studies (Dan and Poo, 1992; Akaaboune et al., 1999; Wan and Poo, 1999; Ouanounou et al., 2016) that suggest synaptic strength at the NMJ is dynamically modulated.

\section{References}

Abraham WC (2008) Metaplasticity: tuning synapses and networks for plasticity. Nat Rev Neurosci 9:387-399.

Abraham WC, Bear MF (1996) Metaplasticity: the plasticity of synaptic plasticity. Trends Neurosci 19:126-130.

Akaaboune M, Culican SM, Turney SG, Lichtman JW (1999) Rapid and reversible effects of activity on acetylcholine receptor density at the neuromuscular junction in vivo. Science 286:503-507.

Baumbauer KM, Huie JR, Hughes AJ, Grau JW (2009) Timing in the absence of supraspinal input: II. Regularly spaced stimulation induces a lasting alteration in spinal function that depends on the NMDA receptor, BDNF release, and protein synthesis. J Neurosci 29:14383-14393.

Berger UV, Carter RE, Coyle JT (1995) The immunocytochemical localization of $\mathrm{N}$-acetylaspartyl glutamate, its hydrolysing enzyme NAALADase, and the NMDAR-1 receptor at a vertebrate neuromuscular junction. Neuroscience 64:847-850. 
Bertuzzi M, Chang W, Ampatzis K (2018) Adult spinal motoneurons change their neurotransmitter phenotype to control locomotion. Proc Natl Acad Sci USA 115:E9926-E9933.

Boulland JL, Qureshi T, Seal RP, Rafiki A, Gundersen V, Bergersen LH, Fremeau RT Jr, Edwards RH, Storm-Mathisen J, Chaudhry FA (2004) Expression of the vesicular glutamate transporters during development indicates the widespread corelease of multiple neurotransmitters. J Comp Neurol 480:264-280.

Brunelli G, Spano P, Barlati S, Guarneri B, Barbon A, Bresciani R, Pizzi M (2005) Glutamatergic reinnervation through peripheral nerve graft dictates assembly of glutamatergic synapses at rat skeletal muscle. Proc Natl Acad Sci USA 102:8752-8757.

Chi SI, Levine JD, Basbaum AI (1993) Effects of injury discharge on the persistent expression of spinal cord fos-like immunoreactivity produced by sciatic nerve transection in the rat. Brain Res 617:220-224.

Colombo MN, Francolini M (2019) Glutamate at the vertebrate neuromuscular junction: from modulation to neurotransmission. Cells 8:996.

Cohen J (1988) Statistical Power Analysis for the Behavioural Sciences, Ed 2. Hillsdale, New Jersey: Lawrence. Erlbaum Associates.

Costigan M, Scholz J, Woolf CJ (2009) Neuropathic pain: a maladaptive response of the nervous system to damage. Annu Rev Neurosci 32:1-32.

Crown ED, Grau JW (2001) Preserving and restoring behavioral potential within the spinal cord using an instrumental training paradigm. J Neurophysiol 86:845-855.

Crown ED, Ferguson AR, Joynes RL, Grau JW (2002a) Instrumental learning within the spinal cord: IV. Induction and retention of the behavioral deficit observed after noncontingent shock. Behav Neurosci 116:1032-1051.

Crown ED, Ferguson AR, Joynes RL, Grau JW (2002b) Instrumental learning within the spinal cord: II. Evidence for central mediation. Physiol Behav 77:259-267.

Dan Y, Poo MM (1992) Hebbian depression of isolated neuromuscular synapses in vitro. Science 256:1570-1573.

Davis JA, Bopp AC, Henwood MK, Baine RE, Cox CC, Grau JW (2020) Pharmacological transection of brain-spinal cord communication blocks pain-induced hemorrhage and locomotor deficits after spinal cord injury. J Neurotrauma 37:1729-1739.

el-Bohy AA, Schrimsher GW, Reier PJ, Goshgarian HG (1998) Quantitative assessment of respiratory function following contusion injury of the cervical spinal cord. Exp Neurol 150:143-152.

Ewald P, Neuhuber WL, Raab M (2006) Vesicular glutamate transporter 1 immunoreactivity in extrinsic and intrinsic innervation of the rat esophagus. Histochem Cell Biol 125:377-395.

Ferguson AR, Crown ED, Grau JW (2006) Nociceptive plasticity inhibits adaptive learning in the spinal cord. Neuroscience 141:421-431.

Ferguson AR, Huie JR, Crown ED, Grau JW (2012a) Central nociceptive sensitization vs. spinal cord training: opposing forms of plasticity that dictate function after complete spinal cord injury. Front Physiol 3:396.

Ferguson AR, Huie JR, Crown ED, Baumbauer KM, Hook MA, Garraway SM, Lee KH, Hoy KC, Grau JW (2012b) Maladaptive spinal plasticity opposes spinal learning and recovery in spinal cord injury. Front Physiol 3:399.

Ferguson AR, Bolding KA, Huie JR, Hook MA, Santillano DR, Miranda RC, Grau JW (2008) Group I metabotropic glutamate receptors control metaplasticity of spinal cord learning through a protein kinase c-dependent mechanism. J Neurosci 28:11939-11949.

Forssberg H (1979) Stumbling corrective reaction: a phase-dependent compensatory reaction during locomotion. J Neurophysiol 42:936-953.

Forssberg H, Grillner S, Rossignol S (1977) Phasic gain control of reflexes from the dorsum of the paw during spinal locomotion. Brain Res 132:121-139.

Francolini M, Brunelli G, Cambianica I, Barlati S, Barbon A, La Via L, Guarneri B, Boroni F, Lanzillotta A, Baiguera C, Ettorre M, Buffelli M, Spano P, Clementi F, Pizzi M (2009) Glutamatergic reinnervation and assembly of glutamatergic synapses in adult rat skeletal muscle occurs at cholinergic endplates. J Neuropathol Exp Neurol 68:1103-1115.

Grau JW (1984) Influence of naloxone on shock-induced freezing and analgesia. Behav Neurosci 98:278-292.

Grau JW (2014) Learning from the spinal cord: how the study of spinal cord plasticity informs our view of learning. Neurobiol Learn Mem 108:155171.

Grau JW, Huang YJ (2018) Metaplasticity within the spinal cord: evidence brain-derived neurotrophic factor (BDNF), tumor necrosis factor (TNF), and alterations in GABA function (ionic plasticity) modulate pain and the capacity to learn. Neurobiol Learn Mem 154:121-135.

Grau JW, Barstow DG, Joynes RL (1998) Instrumental learning within the spinal cord: I. Behavioral properties. Behav Neurosci 112:1366-1386.

Grau JW, Crown ED, Ferguson AR, Washburn SN, Hook MA, Miranda RC (2006) Instrumental learning within the spinal cord: underlying mechanisms and implications for recovery after injury. Behav Cogn Neurosci Rev 5:191-239.

Grau JW, Huie JR, Garraway SM, Hook MA, Crown ED, Baumbauer KM, Lee KH, Hoy KC, Ferguson AR (2012) Impact of behavioral control on the processing of nociceptive stimulation. Front Physiol 3:262.

Grau JW, Huie JR, Lee KH, Hoy KC, Huang YJ, Turtle JD, Strain MM, Baumbauer KM, Miranda RM, Hook MA, Ferguson AR, Garraway SM (2014) Metaplasticity and behavior: how training and inflammation affect plastic potential within the spinal cord and recovery after injury. Front Neural Circuits 8:100.

Grau JW, Baine RE, Bean PA, Davis JA, Fauss GN, Henwood MK, Hudson KE, Johnston DT, Tarbet MM, Strain MM (2020) Learning to promote recovery after spinal cord injury. Exp Neurol 330:113334.

Grozdanovic Z, Gossrau R (1998) Co-localization of nitric oxide synthase I (NOS I) and NMDA receptor subunit 1 (NMDAR-1) at the neuromuscular junction in rat and mouse skeletal muscle. Cell Tissue Res 291: $57-63$.

Herzog E, Landry M, Buhler E, Bouali-Benazzouz R, Legay C, Henderson CE, Nagy F, Dreyfus P, Giros B, El Mestikawy S (2004) Expression of vesicular glutamate transporters, VGLUT1 and VGLUT2, in cholinergic spinal motoneurons. Eur J Neurosci 20:1752-1760.

Hoy KC, Huie JR, Grau JW (2013) AMPA receptor mediated behavioral plasticity in the isolated rat spinal cord. Behav Brain Res 236:319-326.

Huie JR, Baumbauer KM, Lee KH, Bresnahan JC, Beattie MS, Ferguson AR, Grau JW (2012a) Glial tumor necrosis factor alpha (TNF alpha) generates metaplastic inhibition of spinal learning. PLoS One 7:e39751.

Huie JR, Garraway SM, Baumbauer KM, Hoy KC, Beas BS, Montgomery KS, Bizon JL, Grau JW (2012b) Brain-derived neurotrophic factor promotes adaptive plasticity within the spinal cord and mediates the beneficial effects of controllable stimulation. Neuroscience 200:74-90.

Ji RR, Kohno T, Moore KA, Woolf CJ (2003) Central sensitization and LTP: do pain and memory share similar mechanisms? Trends Neurosci 26: 696-705.

Joynes RL, Ferguson AR, Crown ED, Patton BC, Grau JW (2003) Instrumental learning within the spinal cord: V. Evidence the behavioral deficit observed after noncontingent nociceptive stimulation reflects an intraspinal modification. Behav Brain Res 141:159-170.

Joynes RL, Janjua K, Grau JW (2004) Instrumental learning within the spinal cord: VI. The NMDA receptor antagonist, AP5, disrupts the acquisition and maintenance of an acquired flexion response. Behav Brain Res 154:431-438.

Kasimov MR, Fatkhrakhmanova MR, Mukhutdinova KA, Petrov AM (2017) 24S-Hydroxycholesterol enhances synaptic vesicle cycling in the mouse neuromuscular junction: implication of glutamate NMDA receptors and nitric oxide. Neuropharmacology 117:61-73.

Li H, Harlow ML (2014) Individual synaptic vesicles from the electroplaque of Torpedo californica, a classic cholinergic synapse, also contain transporters for glutamate and ATP. Physiol Rep 2:e00206.

Liu GT, Crown ED, Miranda RC, Grau JW (2005) Instrumental learning within the rat spinal cord: Localization of the essential neural circuit. Behav Neurosci 119:538-547.

Malinow R, Malenka RC (2002) AMPA receptor trafficking and synaptic plasticity. Annu Rev Neurosci 25:103-126.

Malomouzh AI, Nurullin LF, Arkhipova SS, Nikolsky EE (2011) NMDA receptors at the endplate of rat skeletal muscles: precise postsynaptic localization. Muscle Nerve 44:987-989.

Mays TA, Sanford JL, Hanada T, Chishti AH, Rafael-Fortney JA (2009) Glutamate receptors localize postsynaptically at neuromuscular junctions in mice. Muscle Nerve 39:343-349.

Nishimaru H, Restrepo CE, Ryge J, Yanagawa Y, Kiehn O (2005) Mammalian motor neurons corelease glutamate and acetylcholine at central synapses. Proc Natl Acad Sci USA 102:5245-5249.

Ouanounou G, Baux G, Bal T (2016) A novel synaptic plasticity rule explains homeostasis of neuromuscular transmission. Elife 5:e12190.

Personius KE, Slusher BS, Udin SB (2016) Neuromuscular NMDA receptors modulate developmental synapse elimination. J Neurosci 36:8783-8789. 
Petrov KA, Malomouzh AI, Kovyazina IV, Krejci E, Nikitashina AD, Proskurina SE, Zobov VV, Nikolsky EE (2013) Regulation of acetylcholinesterase activity by nitric oxide in rat neuromuscular junction via Nmethyl-D-aspartate receptor activation. Eur J Neurosci 37:181-189.

Pinard A, Robitaille R (2008) Nitric oxide dependence of glutamate-mediated modulation at a vertebrate neuromuscular junction. Eur J Neurosci 28:577-587.

Pizzi M, Brunelli G, Barlati S, Spano P (2006) Glutamatergic innervation of rat skeletal muscle by supraspinal neurons: a new paradigm in spinal cord injury repair. Curr Opin Neurobiol 16:323-328.

Pullen AH (1977) The distribution and relative sizes of three histochemical fibre types in the rat tibialis anterior muscle. J Anat 123:1-19.

Ribera J, Marsal J, Casanovas A, Hukkanen M, Tarabal O, Esquerda JE (1998) Nitric oxide synthase in rat neuromuscular junctions and in nerve terminals of Torpedo electric organ: its role as regulator of acetylcholine release. J Neurosci Res 51:90-102.

Sanes JR, Lichtman JW (1999) Development of the vertebrate neuromuscular junction. Annu Rev Neurosci 22:389-442.

Sanes JR, Lichtman JW (2001) Induction, assembly, maturation and maintenance of a postsynaptic apparatus. Nat Rev Neurosci 2:791-805.
Schmidt MV, Abraham WC, Maroun M, Stork O, Richter-Levin G (2013) Stress-induced metaplasticity: from synapses to behavior. Neuroscience 250:112-120.

Slater CR (2015) The functional organization of motor nerve terminals. Prog Neurobiol 134:55-103.

Tabone CJ, Ramaswami M (2012) Is NMDA receptor-coincidence detection required for learning and memory? Neuron 74:767-769.

Vichaya EG, Baumbauer KM, Carcoba LM, Grau JW, Meagher MW (2009) Spinal glia modulate both adaptive and pathological processes. Brain Behav Immun 23:969-976.

Waerhaug O, Ottersen OP (1993) Demonstration of glutamate-like immunoreactivity at rat neuromuscular junctions by quantitative electron microscopic immunocytochemistry. Anat Embryol (Berl) 188:501-513.

Walters ET, Byrne JH (1983) Associative conditioning of single sensory neurons suggests a cellular mechanism for learning. Science 219: 405-408.

Wan J, Poo M (1999) Activity-induced potentiation of developing neuromuscular synapses. Science 285:1725-1728.

Wood SJ, Slater CR (2001) Safety factor at the neuromuscular junction. Prog Neurobiol 64:393-429. 